\title{
Disruption of astrocyte-neuron cholesterol cross talk affects neuronal function in Huntington's disease
}

\author{
M Valenza ${ }^{1,3}$, M Marullo $^{1,3}$, E Di Paolo', E Cesana ${ }^{2}$, C Zuccato ${ }^{1}$, G Biella ${ }^{2}$ and E Cattaneo*,1
}

In the adult brain, neurons require local cholesterol production, which is supplied by astrocytes through apoE-containing lipoproteins. In Huntington's disease (HD), such cholesterol biosynthesis in the brain is severely reduced. Here we show that this defect, occurring in astrocytes, is detrimental for HD neurons. Astrocytes bearing the huntingtin protein containing increasing CAG repeats secreted less apoE-lipoprotein-bound cholesterol in the medium. Conditioned media from HD astrocytes and lipoprotein-depleted conditioned media from wild-type (wt) astrocytes were equally detrimental in a neurite outgrowth assay and did not support synaptic activity in HD neurons, compared with conditions of cholesterol supplementation or conditioned media from wt astrocytes. Molecular perturbation of cholesterol biosynthesis and efflux in astrocytes caused similarly altered astrocyteneuron cross talk, whereas enhancement of glial SREBP2 and ABCA1 function reversed the aspects of neuronal dysfunction in HD. These findings indicate that astrocyte-mediated cholesterol homeostasis could be a potential therapeutic target to ameliorate neuronal dysfunction in HD.

Cell Death and Differentiation (2015) 22, 690-702; doi:10.1038/cdd.2014.162; published online 10 October 2014

Huntington's disease (HD) is an adult-onset neurodegenerative disorder characterized by cell loss mainly in the striatum and cortex. Its pathophysiology is linked to an expanded CAG repeat in the IT-15 gene, which leads to an elongated polyQ tract in huntingtin (HTT) protein. No disease-modifying treatment is available for HD and novel pathophysiological insights and therapeutic strategies are needed. ${ }^{1}$

Lipids are vital to brain health and function. Accordingly, the brain has a local source of cholesterol, ${ }^{2}$ and a breakdown of cholesterol synthesis causes brain malformations and impaired cognitive function. ${ }^{3,4}$ Cholesterol metabolism is disrupted in $\mathrm{HD}^{5,6}$ as revealed by transcriptional, biochemical, and mass spectrometry analyses in HD rodent models. ${ }^{7,8}$ This dysregulation is linked to a specific action of mutant HTT on sterol-regulatory-element-binding proteins (SREBPs) and on its target genes, whose reduced transcription leads to lower brain cholesterol levels. ${ }^{7}$ In HD humans, brain cholesterol homeostasis is affected since pre-symptomatic stages, as determined by measurement of the brain-specific cholesterol catabolite 24-S-hydroxy-cholesterol (24OHC). ${ }^{9,10}$ However, it remains unclear how reduced brain cholesterol would become pathological for HD neurons.

In adulthood, astrocytes produce cholesterol, which is secreted as a complex with apolipoprotein (apo) E lipoproteins and delivered to neurons. ${ }^{11,12}$ Mutant HTT is expressed in glial cells, ${ }^{13,14}$ and transgenic mice overexpressing mutant HTT in astrocytes show age-dependent neurological symptoms. ${ }^{15,16}$ Additionally, primary astrocytes overexpressing full-length human mutant HTT show reduced mRNA levels of cholesterol biosynthetic genes, along with impaired cellular production and secretion of apoE. ${ }^{8}$

Here we employed molecular and cellular tools to test the impact of cholesterol perturbation between astrocytes and neurons in HD. Reduced secretion of cholesterol bound to apoE lipoproteins by HD astrocytes negatively influenced neurite outgrowth and neuronal synaptic properties. Furthermore, gain-of-function experiments revealed that this non-cellautonomous mechanism can be overridden through molecular enhancement of cholesterol biosynthesis in HD astrocytes.

\section{Results}

Astrocytes bearing mutant HTT protein display cholesterol dysfunction. To test whether and how cholesterol dysfunction in HD astrocytes impacted neuronal function, we took advantage of neural stem (NS) cells that are capable of differentiating into neurons or astrocytes depending on culture conditions $^{17}$ (Supplementary Figures $1 \mathrm{a}$ and $\mathrm{e}$ ). We first found that mRNA levels of hydroxyl-methyl-glutarylCoA reductase ( $h m g c r)$ were similar between wild-type (wt) and HD NS cells under self-renewal conditions, but were severely reduced in $\mathrm{GFAP}^{+} \mathrm{NS}$-derived astrocytes carrying mutant HTT with 140 CAG repeats (Q140/7 astrocytes) compared with wt NS-derived astrocytes (Q7/7 astrocytes) (Figure 1a). Accordingly, following $6-8 \mathrm{~h}$ of pre-incubation with serum-free medium, the Q140/7 astrocytes secreted less

${ }^{1}$ Department of Biosciences and Centre for Stem Cell Research, Università degli Studi di Milano, Milano, Italy and ${ }^{2}$ Department of Biology and Biotechnology, Università degli Studi di Pavia, Pavia, Italy

*Corresponding author: E Cattaneo, Department of Biosciences and Centre for Stem Cell Research, Università degli Studi di Milano, Via Viotti 3/5, Milan, 20133, Italy. Tel: +39 02 50325830; Fax: +39 02 50325843; E-mail: elena.cattaneo@ unimi.it

${ }^{3}$ These authors contributed equally to this work

Abbreviations: 24OHC, 24-S-hydroxy-cholesterol; Bsn, Bassoon; CAG, cytosine-adenine-guanine; fdft1, farnesyl-diphosphate farnesyltransferase; GCM, glialconditioned medium; HD, Huntington's disease; Hmgcr, hydroxyl-methyl-glutaryl-CoA reductase; HTT, huntingtin; LRP1, LDL receptor-related protein; NS, neural stem; SRE, sterol regulatory element; SREBPs, sterol regulatory element-binding proteins; $\mathrm{Wt}$, wild-type

Received 20.5.14; revised 13.8.14; accepted 03.9.14; Edited by M Piacentini; published online 10.10.14 
apoE in the media compared with Q7/7 astrocytes (Figure 1b). ApoE level was also significantly decreased in the media from an allelic series of Hdh CAG knock-in NS cells carrying 50 or 111 CAG repeats (Q50/7 and Q111/7 astrocytes) in a CAG length-dependent manner (Q7/7>Q50/7>Q111/7) (Figure 1c). ${ }^{17}$ HTT-depleted astrocytes displayed the same reduction in apoE level as in Q111/7 (Figure 1c), supporting the hypothesis that normal HTT has a role in cholesterol synthesis. ${ }^{18}$ Furthermore, primary astrocytes from a transgenic HD mouse model (R6/2; Supplementary Figure 1f) secreted lower apoE levels compared with primary wt astrocytes (Figure 1d). Quantitative assays confirmed that concentrations of apoE (Figure 1e) and cholesterol (Figure 1f) were significantly reduced in conditioned media from Q140/7 astrocytes and from primary R6/2 astrocytes compared with their respective controls. The impairment in apoE release is likely due to reduced synthesis of intracellular apoE as indicated by reduced mRNA and protein levels in YAC128 astrocytes $^{8}$ and in R6/2 and HD NS-derived astrocytes (Supplementary Figure 1g).

Although apoE level was reduced in the media from R6/2 astrocytes, the sizes of apoE lipoproteins, as judged by nondenaturing gel electrophoresis, were similar in the media from the control and HD cultures (Figure $1 \mathrm{~g}$ ). This finding suggests that the total content of cholesterol bound to apoE lipoproteins secreted by HD astrocytes was lower because of reduced apoE level and not as a consequence of inefficient lipoprotein lipidation. Together, these results indicate that HD astrocytes produce and secrete less cholesterol bound to apoE lipoproteins in vitro, with possible consequences for neuronal function.

Neurite outgrowth is reduced in HD neurons and is rescued by exogenous cholesterol. Upon exposure to a pan-neuronal differentiation protocol, NS cells can be converted into generic MAP2 ${ }^{+}$neurons. ${ }^{17,19}$ Here, we first differentiated wt and HD NS cell lines towards neurons, and after 7 days, the cells were fixed and immunostained with an anti-MAP2 antibody to visualize the neurites. Not surprisingly, neurite outgrowth was reduced in Q140/7 neurons compared with Q7/7 neurons, as shown by MAP2 staining (Figure $1 \mathrm{~h}$ ) and relative quantification using an automated neurite tracing $\left(\right.$ NeuriteTracer ${ }^{20}$ ) (Figure 1i). Flow cytometric analysis also showed a reduced number of MAP2-positive cells in Q140/7 (28.3\%) compared with Q7/7 neurons (44.1\%; Supplementary Figure 2a). Notably, addition of cholesterol improved neurite outgrowth in Q140/7 neurons (Figures $1 \mathrm{~h}$ and i) with maximal effect at $7-10 \mu \mathrm{g} / \mathrm{ml}$ (Figure 1j). Above this concentration, cholesterol failed to promote neurite outgrowth, as depicted by the bell-shaped curves for Q140/7 neurons (Figure 1j) and, to a lesser extent, for Q7/7 neurons (Supplementary Figure 2b). In the same experimental paradigm, cholesterol treatment did not influence cell survival/proliferation in control or HD neurons (Supplementary Figure 2c). Overall, these results highlight a relationship between neurite outgrowth and cholesterol administration, demonstrating that exogenous cholesterol application is beneficial to HD neurons in vitro.
Glial-conditioned medium from HD astrocytes does not support neurite outgrowth in HD neurons. To test whether glial-conditioned media (GCM) influenced HD neurons, we quantified neurite outgrowth following exposure to GCM from wt and HD astrocytes. First, GCM from primary and NSderived astrocytes were prepared (see Method section) and used to supplement the regular growth medium of Q140/7 neurons, starting from day 4 of neuronal differentiation. As observed for exogenous cholesterol administration, GCM from primary wt astrocytes (GCM wt) or from Q7/7 astrocytes (GCM Q7/7) promoted neurite outgrowth in Q140/7 neurons with respect to the cells under glial-free condition (not treated, NT) (Figures $2 a$ and b). Conversely, GCM from primary astrocytes generated from R6/2 mice (GCM R6/2), or from Q50/7 or Q140/7 astrocytes (GCM Q50/7 and GCM Q140/7), did not promote neurite outgrowth (Figures $2 a$ and b). Not surprisingly, all GCMs (except for R6/2) performed better than NT, although the difference was not significant, suggesting that other glial components, not affected in the HD condition, contribute to neurite outgrowth.

To test whether lipoproteins in the GCM were responsible for promoting neurite outgrowth, we cultured Q140/7 neurons with lipoprotein-depleted GCM from wt astrocytes $\left(\mathrm{GCM}_{\mathrm{wt}}\right.$ delip) or GCM from primary astrocytes generated from apoE knock-out mice (GCM apoE ${ }^{-1}$ ). Neither GCM stimulated neurite outgrowth in Q140/7 neurons (Supplementary Figure 3a,b). Notably, neither GCM contained a detectable apoE level (Supplementary Figure 3c) whereas GCM apoE ${ }^{-/-}$contained higher levels of apoD (Supplementary Figure 3d), another lipoprotein expressed in the brain, which is increased in apoE ${ }^{-1-}$ mice to carry out some of the apoE-dependent functions. ${ }^{21}$ These data indicate that apoE lipoproteins in GCM are key factors in stimulating neurite outgrowth by supplying cholesterol to HD neurons.

\section{Genetic perturbation of astrocytic cholesterol production} and release influences neurite outgrowth in HD neurons. To further test the impact of cholesterol perturbation on neurite outgrowth in HD neurons, we performed gain- and loss-of-function experiments. We first silenced abca1, the gene encoding ATP-binding cassette transporter ABCA1, the major regulator of cellular cholesterol homeostasis. Transfection of wt primary astrocytes with small interfering RNAs (siRNA) against abca reduced the level of abca1 mRNA (abca-i) by $40 \%$ compared with wt astrocytes transfected with the scrambled siRNA control (scrambled) (Figure 3a). This led to a $50 \%$ decrease of apoE levels secreted in the GCM $\left(\mathrm{GCM}_{\mathrm{wt}}\right.$ abca-i) compared with GCM from scrambled astrocytes $\left(\mathrm{GCM}_{\mathrm{wt}} \mathrm{scrambled)}\right.$ (Figure 3b). Importantly, neurite outgrowth was severely impaired when Q140/7 neurons were cultured with $\mathrm{GCM}_{\text {wt }}$ abca-i, but not with $\mathrm{GCM}_{\text {wt }}$ scrambled (Figures $3 c$ and $d$ ). Overexpression of $A B C A 1$ in $R 6 / 2$ astrocytes (Figure $3 e$ ) did not lead to increased apoE level in the GCM $\left(\mathrm{GCM}_{\mathrm{R} 6 / 2} \mathrm{ABCA} 1\right)$ compared with $\mathrm{GCM}_{\mathrm{R} 6 / 2}$ pcDNA (Figure 3f, left), in agreement with the evidence that greater than sixfold overexpression of $A B C A 1$ in the mouse brain is required to alter apoE levels. ${ }^{22}$ However, ABCA1 overexpression stimulated R6/2 astrocytes to release larger apoE lipoproteins into the medium 
(Figure 3f, right, arrows), suggesting increased lipidation. When HD neurons were cultured with GCM from R6/2 astrocytes overexpressing $A B C A 1\left(\mathrm{GCM}_{\mathrm{R} 6 / 2} \mathrm{ABCA} 1\right)$, we observed stimulated neurite outgrowth compared with untreated $\mathrm{HD}$ neurons or treated with $\mathrm{GCM}_{\mathrm{R} / 2 / 2} \mathrm{pCDNA}$ (Figures $3 g$ and $h$ ). a

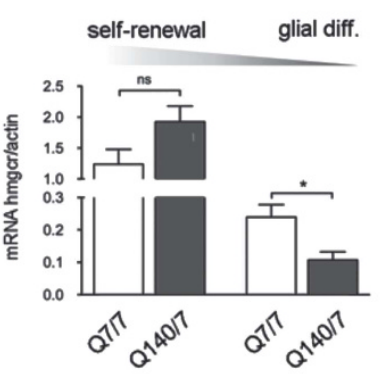

e

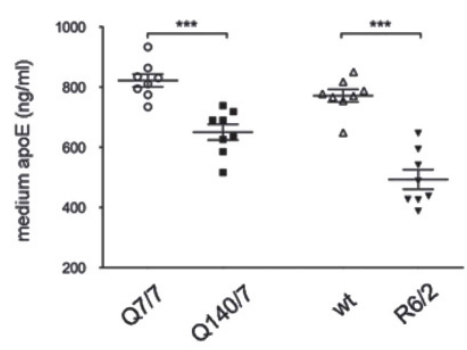

b

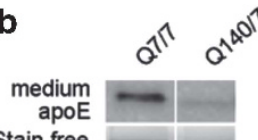
Stain free

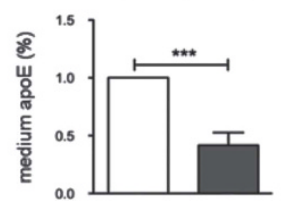

$\mathbf{f}$

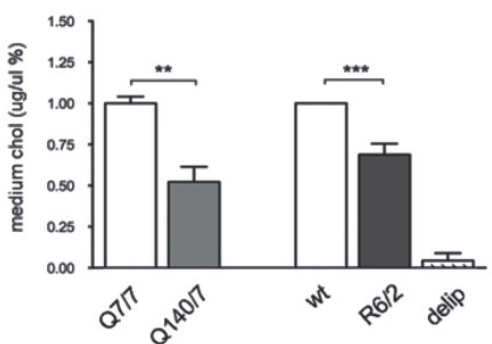

d

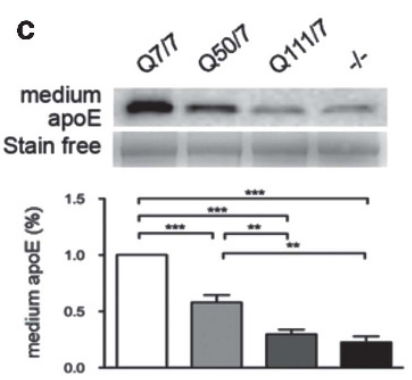

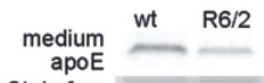

Stain free

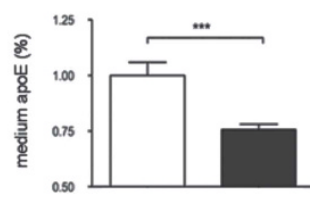

h

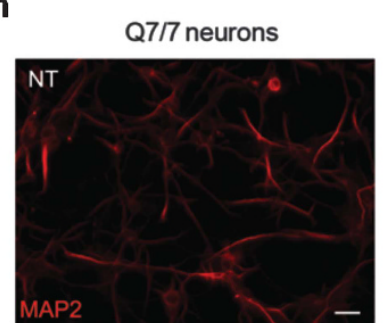

Q140/7 neurons

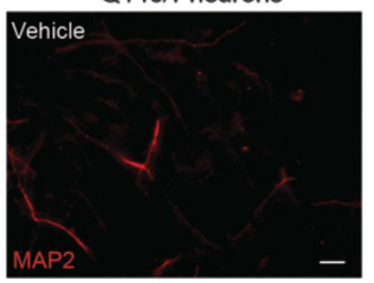

Q140/7 neurons

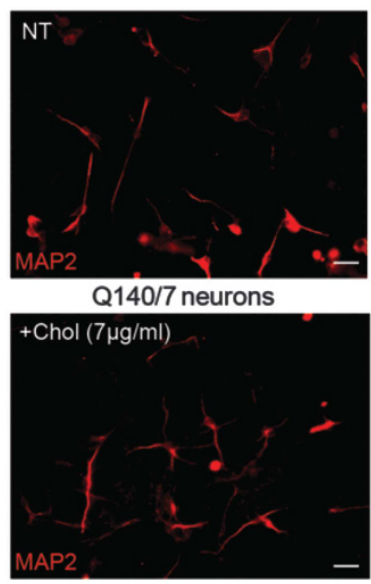

i

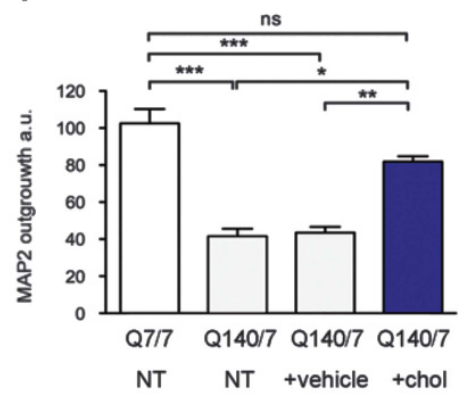

j

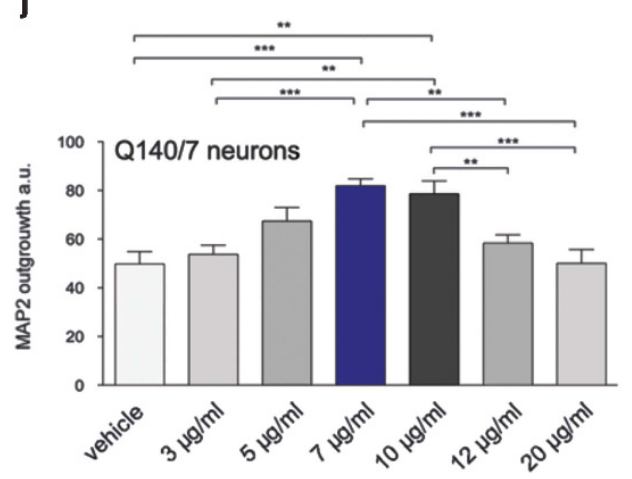


We also silenced in HD neurons the gene encoding for LDL receptor-related protein (LRP1), the major neuronal apoE receptor $^{23}$ (Supplementary Figure 4a). As expected, GCM wt and GCM Q7/7 both promoted neurite outgrowth in HD neurons transfected with the scrambled siRNA compared with the same NT neurons and both failed to promote neurite outgrowth when Irp1 had been silenced (Supplementary Figures $4 b$ and c). However, the reduced Irp1 mRNA level is not mechanistically linked to cholesterol dysfunction, because the Irp1 mRNA level was indistinguishable between wt and $\mathrm{R} 6 / 2$ brains at pre- and late-symptomatic stages (Supplementary Figure 4d). These data indicate that the cholesterol uptake system is intact in HD neurons, but a defect in cholesterol synthesis reduces the amount of astrocytederived cholesterol available for neuronal function.

SREBP2 is the master transcription factor that activates the expression of nearly all cholesterol biosynthesis genes by binding to the sterol regulatory element (SRE) in target gene promoters. Previous studies reported that SREBP activity and its nuclear translocation were reduced in an inducible cell model of HD and in R6/2 brains. ${ }^{7}$ Reduced nuclear levels of the N-terminal active fragment of SREBP2 (Nt-BP2) were also found in R6/2 astrocytes compared with wt astrocytes (Supplementary Figure 5a) and mRNA levels of SREBP2, but not of SREBP1, were decreased in HD NS-derived astrocytes compared with controls (Supplementary Figure $5 b)$. Notably, a SRE sequence is present in the promoter region of SREBP2, ${ }^{24}$ suggesting that reduced SREBP2 activation may in turn affect srebp2 gene expression. Knockdown of srebp2 expression in primary wt astrocytes (Figure 4a) led to decreased apoE levels in the GCM $\left(\mathrm{GCM}_{\mathrm{wt}} \mathrm{srebp2-i)}\right.$ compared with in GCM from wt astrocytes nucleofected with the scrambled-siRNA (GCM ${ }_{\mathrm{wt}} \mathrm{scrambled)}$ (Figure 4b). The presence of $\mathrm{GCM}_{\mathrm{wt}}$ srebp2-i elicited a robust phenotype in HD neurons, which included the inability to promote neurite outgrowth with respect to $\mathrm{GCM}_{\mathrm{wt}} \mathrm{scrambled}$ (Figures 4c and d). In contrast, overexpression of Nt-BP2 in primary $R 6 / 2$ astrocytes stimulated an increase of mRNA levels of hmgcr and farnesyl-diphosphate farnesyltransferase (fdft1), followed by an increase in abca1 mRNA at $67 \mathrm{~h}$ of nucleofection (Figure $4 \mathrm{e}$ ). At this time point, the relative GCM consisted of larger apoE lipoproteins (Figure 4f). Similarly to ABCA1 overexpression, we found that neurite outgrowth was increased when Q140/7 neurons were cultured with $\mathrm{GCM}_{\mathrm{R} 6 / 2} \mathrm{Nt}-\mathrm{BP} 2$ compared with NT neurons or to neurons cultured with $\mathrm{GCM}_{\mathrm{R} 6 / 2}$ pcDNA (Figures $4 \mathrm{~g}$ and h). Similar findings were obtained with Q50/7 neurons (Supplementary
Figure 6). ApoE, albeit at low levels, is essential along with newly cholesterol to rescue neuritic defects in HD neurons. Indeed, GCM from apoE ${ }^{-/-}$astrocytes overexpressing NtBP2 failed to rescue neurite outgrowth in HD neurons (Supplementary Figure 7).

Together, these data indicate that cholesterol biosynthesis modulation in HD astrocytes influences the secretion of cholesterol bound to apoE-containing lipoproteins and neurite outgrowth in HD neurons.

GCM from HD astrocytes reduces synaptic properties in HD neurons. As cholesterol secreted by astrocytes is crucial for synaptic formation and maintenance, ${ }^{25}$ we looked at the synaptic-promoting capacities of GCM wt and GCM HD in HD neurons. We cultured striatal or cortical primary neurons of $\mathrm{R} 6 / 2$ mice in the presence of GCM wt (from primary wt astrocytes) or GCM HD (from primary R6/2 astrocytes), starting from 4 days in vitro (div). At 11 div, we counted the number of bona fide synaptic contacts by double-staining the cultures for the pre-synaptic scaffold Bassoon (Bsn) and for vGLUT2 or GABA. We then counted the number of Bsn/ GABA or Bsn/vGLUT2 puncta to define glutamatergic or GABAergic synapses, respectively (Figures $5 a$ and b). Under glial-free conditions, HD striatal neurons displayed significantly reduced numbers of bona fide synaptic contacts compared with wt neurons (Figures 5c). The presence of GCM wt reversed the defect in HD striatal neurons, and increased the number of synaptic contacts in HD cortical neurons (Figures 5c and d). However, GCM HD and $\mathrm{GCM}_{\mathrm{wt}}$ delip failed to do so. The ability of GCM wt, but not of GCM HD or $\mathrm{GCM}_{\mathrm{wt}}$ delip, to positively influence this parameter in HD neurons was also confirmed by western blot analyses showing increased levels of post-synaptic density protein PSD95 in HD neurons only when exposed to GCM wt (Figures $5 e$ and f).

GCM HD impedes synaptic activity in HD neurons. We then assayed the electrophysiological properties of HD neurons through whole-cell patch-clamp technique after the different treatments. Most of the recorded primary striatal neurons from R6/2 mice (Figure 6a) exposed to GCM wt or GCM HD were functionally mature, as demonstrated by the presence of consistent families of inward and outward currents (mediated by voltage-dependent sodium and potassium channels, respectively) and by their ability to generate overshooting action potentials in response to suitable current step injections (Figures $6 \mathrm{~b}$ and $\mathrm{c}$ ). To test whether the

Figure 1 Cholesterol dysfunction in HD astrocytes and rescue of neuritic defects in HD neurons by cholesterol. (a) HmgcrmRNA levels in Hdh ${ }^{\text {Q7/7 }}$ and Hdh ${ }^{\text {Q140/7 }}$ NS cell lines (herein, Q7/7 and Q140/7) during self-renewal and after glial differentiation. Beta-actin was used as the housekeeping gene. The graph shows the mean \pm S.E.M. of three realtime PCR runs from two independent differentiations. (b-d) Representative western blot and densitometry for apoE levels in GCM (medium apoE) from Q7/7 and Q140/7 astrocytes (b), from allelic series of NS-derived astrocytes (Q7/7, Q50/7, and Q111/7) and HTT-depleted astrocytes (c), and from primary wt and R6/2 astrocytes (d). Stain-free imaging (Bio-Rad) was used as a loading control and for normalization. Graph shows mean \% above control \pm S.E.M. for six independent experiments. (e and f) ApoE quantification by ELISA assay (e) and cholesterol quantification by enzymatic assay (f) in a subset of GCMs $(n=3-8)$. The graphs show the mean absolute values (e) or \% above control (f) \pm S.E.M. (g) Representative western blot under non-denaturing conditions for evaluating size of apoE-containing lipoproteins. (h and i) Representative immunofluorescence staining with an antibody against MAP2 (h), and neurite outgrowth quantification (i) of Q7/7 and Q140/7 neurons under glial-free conditions (NT or vehicle) or in the presence of cholesterol (+ chol). The graph in (i) shows the mean of an arbitrary value (a.u.) \pm S.E.M. from one experiment in which 10 fields were analyzed for each condition. Similar results were obtained with four other independent differentiations. (j) Neurite outgrowth quantification in Q140/7 neurons under glial-free conditions (vehicle) or in the presence of increasing doses of cholesterol $(3,5,7,10,12$, and $20 \mu \mathrm{g} / \mathrm{ml})$ immunostained for MAP2. The graph shows the mean (a.u.) \pm S.E.M. of 10 fields for each condition. Statistics: two-tailed unpaired $t$-test $(\mathbf{a}, \mathbf{b}, \mathbf{d}, \mathbf{e}$, and $\mathbf{f})$ or one-way ANOVA, Newman-Keuls multiple-comparison post test $(\mathbf{c}, \mathbf{i}$, and $\mathbf{j})$. ${ }^{*} P<0.05 ;{ }^{* *} P<0.01$; ${ }^{* * *} P<0.001$. See Supplementary Figure 9 for full-length pictures of the blots shown in $\mathbf{b}, \mathbf{c}$, and $\mathbf{d}$ 
a

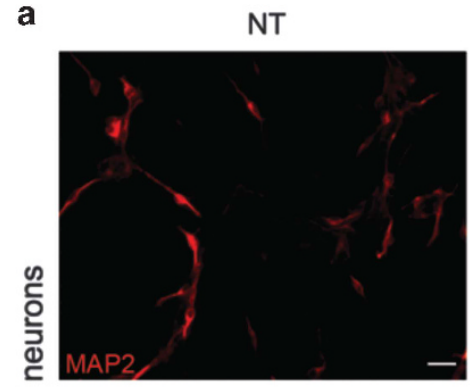

ริำ

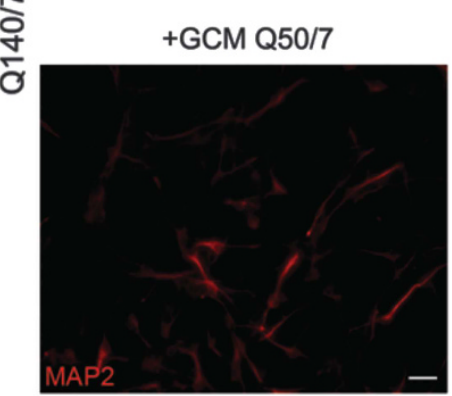

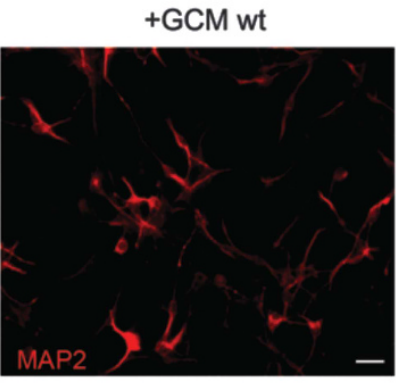

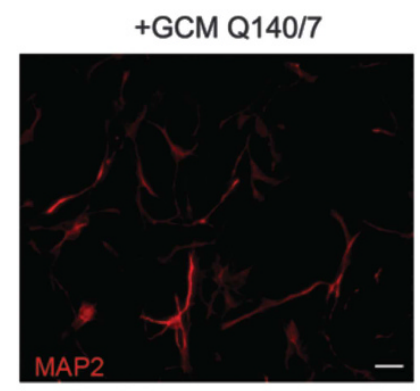

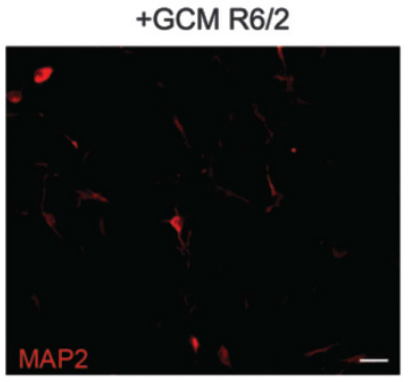

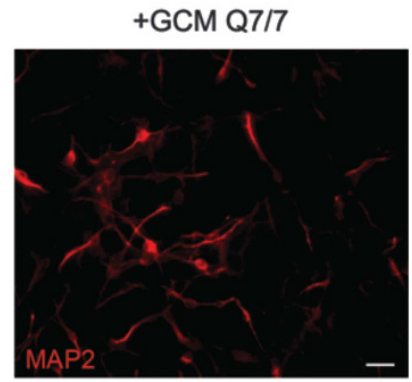

b

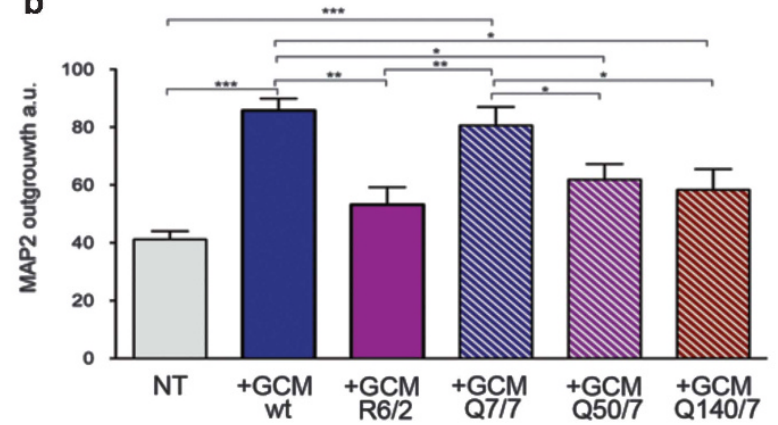

Figure 2 GCM from R6/2 astrocytes does not promote neurite outgrowth in HD NS-derived neurons. (a) Representative immunofluorescence staining for MAP2 in Q140/7 neurons under glial-free conditions (NT) or in the presence of GCM from primary astrocytes from wt mice (+GCM wt) or from R6/2 mice (+GCM R6/2), or GCM from Q7/7 (+GCM Q7/7), Q50/7 (+GCM Q50/7), and Q140/7 (+GCM Q140/7) NS-derived astrocytes. Scale bars: $25 \mu \mathrm{m}$. (b) Relative neurite outgrowth quantification. The graph shows the mean (a.u.) \pm S.E.M. of one experiment in which 10 fields were analyzed for each condition. Similar results were obtained in two other independent differentiations. Statistics: one-way ANOVA, Newman-Keuls multiple-comparison post test (b). ${ }^{*} P<0.05 ;{ }^{* *} P<0.01 ;{ }^{* * \star} P<0.001$

reduced cholesterol complexed to apoE lipoproteins in GCM HD had an impact on synaptic activity in HD neurons, we performed whole-cell recordings at 9-12 div, following incubation with GCM wt or GCM HD for 5-6 days. HD striatal neurons exhibited increased input resistance (Rin) under glial-free conditions compared with wt neurons. Importantly, exogenous cholesterol administration in the presence of GCM HD restored Rin in HD neurons to wt level (Figures 6d). Although the difference between HD and wt neurons in basal conditions was not statistically significant, application of neither GCM wt or GCM HD in HD neurons led to a significant increase in the membrane capacitance $(\mathrm{Cm})$, a parameter directly correlated with cell size (Figure 6e). These results suggest that exogenous cholesterol may influence input resistance, but that other glial-derived molecules might influence the charge storing in HD neurons.

Finally, we tested the capability of the cells to develop in vitro a functional synaptic network, by analyzing post-synaptic activity (Figures $6 f$ and i). Under NT conditions, only 4 out of 27 HD neurons (14.8\%) produced post-synaptic currents compared with 10 out of 22 wt neurons (45.4\%), suggesting that HD neurons had lower spontaneous activity (Figure $6 \mathrm{~g}$ ), consistently with a loss of synaptic contacts and a reduced post-synaptic expression. Notably, 15 out of 22 (68.2\%) of HD neurons showed spontaneous synaptic activity in the presence of GCM wt, and 9 out of $28(32.1 \%)$ in the presence of GCM HD. Cholesterol administration to HD neurons, even in the presence of GCM HD, stimulated spontaneous activity in 7 out of $10(70 \%)$ of the recorded cells (Figure $6 \mathrm{~g}$ ), even with a higher mean frequency $(2.475 \pm 0.99 \mathrm{~Hz})$ compared with untreated HD cells $(0.42 \pm 0.15 \mathrm{~Hz} ; P=0.0238)$ or in the presence of GCM HD $(0.29 \pm 0.06 \mathrm{~Hz} ; P=0.002)$ (Figure 6h). Additionally, the mean amplitude was significantly increased in the presence of GCM wt compared with GCM HD $(34.7 \pm 4.7$ $\mathrm{pA}$ versus $17.30 \pm 4.47 \mathrm{pA} ; P=0.017)$, even in the presence of exogenous cholesterol $(20.93 \pm 4.1 \mathrm{pA})$ (Figure 6i), suggesting that other glial molecules might influence this parameter in HD neurons. These findings indicate that a reduced supply of glial-derived cholesterol affects synaptic activity in HD neurons and this may be reversed by cholesterol supplementation.

\section{Discussion}

Astrocytes, the major cellular component of the $\mathrm{CNS},{ }^{26}$ supply neurons with energy metabolites, growth factors, neurotransmitter recycling functions, and structural support. Cholesterol and glial-conditioned medium have a well-documented role in promoting axonal outgrowth in vitro under physiological conditions. Glial-derived lipoproteins containing cholesterol stimulate axon growth ${ }^{27}$ and transcription of genes involved in dendrite and synapse development ${ }^{28}$ in primary rat retinal ganglion cells; their co-culture with glial cells enhances synaptic activity and neurite outgrowth and branching in Purkinje neurons, ${ }^{29}$ and cholesterol deficiency inhibits dendrite outgrowth in primary cortical and hippocampal neurons. ${ }^{30}$ Impairments in astrocytic function are increasingly recognized as a culprit in neuronal dysfunction in neurodegenerative diseases, ${ }^{31}$ such as amyotrophic lateral sclerosis, $^{32}$ Rett's syndrome, ${ }^{33,34}$ lysosomal disorders, ${ }^{35}$ Alzheimer's disease, ${ }^{36}$ and HD. ${ }^{16}$ Accordingly, astrocyte kir4. 1 ion channel deficits contribute to neuronal dysfunction in 


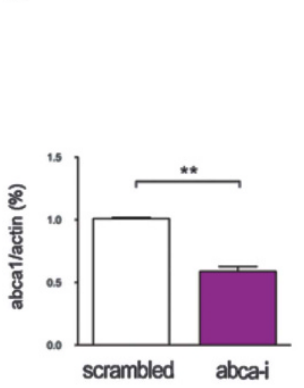

C

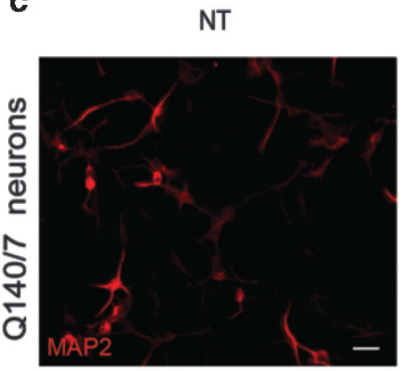

medium apoE

Stain free

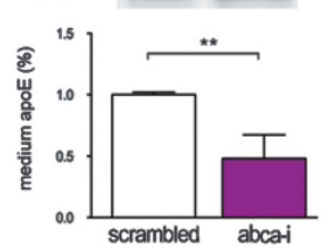

-

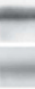

GCM wt
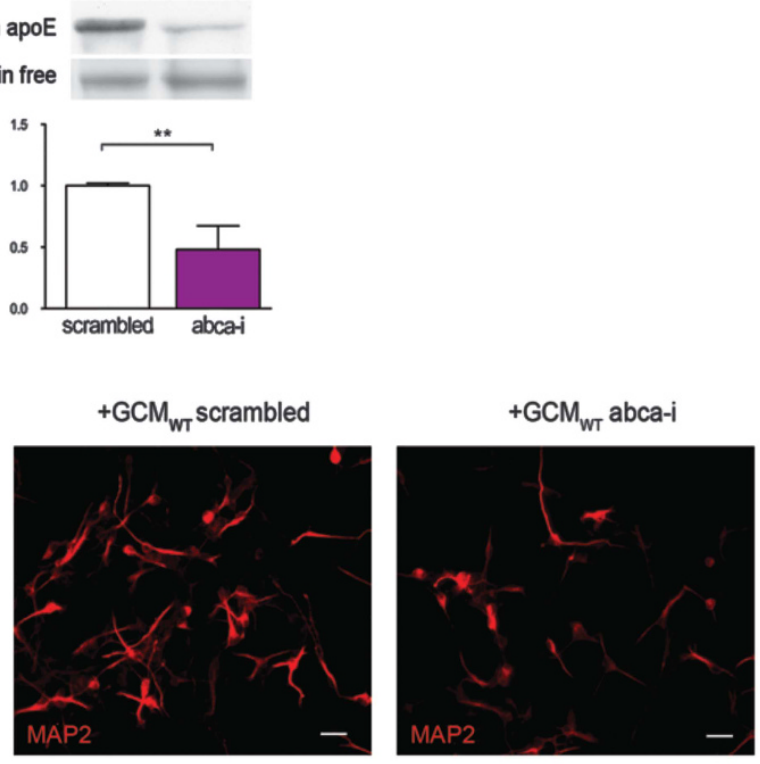

d

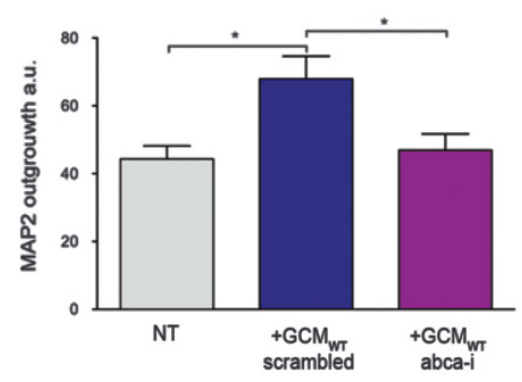

e

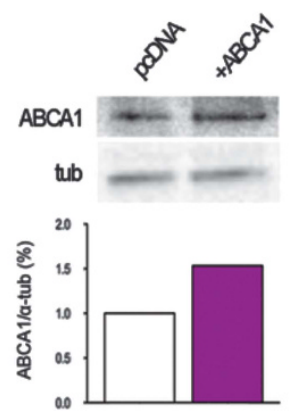

g

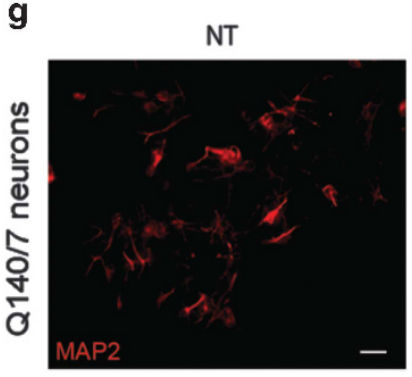

f
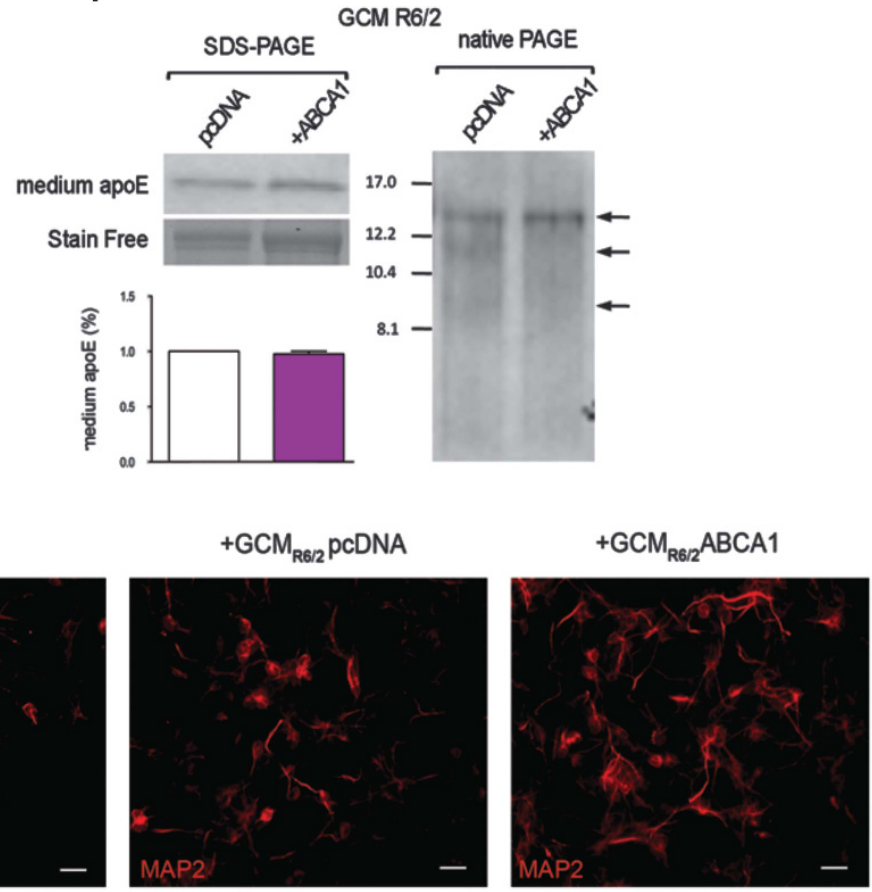

h

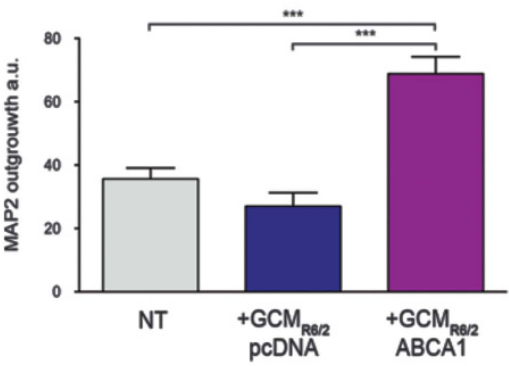

Figure 3 Genetic modulation of ABCA1 in astrocytes influences neurite outgrowth in HD neurons. (a) mRNA levels of abca1 in primary wt astrocytes after transfection with siRNA against abca1 (abca-i) compared with the same cells transfected with scramble siRNA as a control (scrambled). (b) ApoE levels released in the corresponding GCMs.

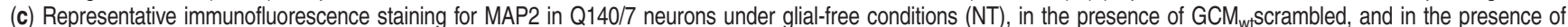

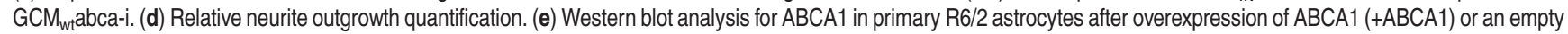
vector (pcDNA). (f) Relative apoE levels (left) and sizes (right) in the corresponding GCM. (g) Representative immunofluorescence staining for MAP2 in Q140/7 neurons under glial-free conditions (NT), in the presence of $\mathrm{GCM}_{\mathrm{R} / 2 / 2} \mathrm{pCDNA}$, and in the presence of $\mathrm{GCM}_{\mathrm{R} / 2 / 2} \mathrm{ABCA1}$. (h) Relative neurite outgrowth quantification. Graphs in $\mathbf{a}, \mathbf{b}, \mathbf{e}$, and $\mathbf{f}$ show the mean \% above relative controls \pm S.E.M. Scale bars: $25 \mu \mathrm{m}$. Graphs in $\mathbf{d}$ and $\mathbf{h}$ show the mean (a.u.) \pm S.E.M. of one experiment in which 10 fields were analyzed for each condition. Similar results were obtained in two (d) and three (h) other independent differentiations. Statistics: two-tailed unpaired t-test (a and $\mathbf{b})$ or one-way ANOVA with Newman-Keuls multiple-comparison post test ( $\mathbf{d}$ and $\mathbf{h}$ ). ${ }^{*} P<0.05 ;{ }^{* \star} P<0.01$; ${ }^{* \star \star} P<0.001$. The samples in $\mathbf{b}$ were run together with $\mathrm{GCM}_{\mathrm{wt}} \mathrm{srebp2-i}$ shown in Figure $4 \mathrm{~b}$. The control sample $\left(\mathrm{GCM}_{\mathrm{wt}} \mathrm{scrambled}\right)$ shown in $\mathbf{b}$ and in Figure $4 \mathbf{b}$ is the same. See Supplementary Figure $\mathbf{9}$ for full-length pictures of the blots shown in $\mathbf{b}, \mathbf{e}$, and $\mathbf{f}$ 
b

\section{GCM wt}

medium apoE

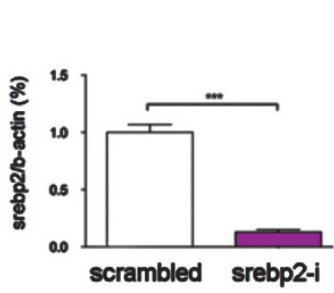

Stain free

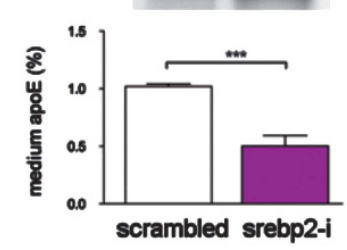

。
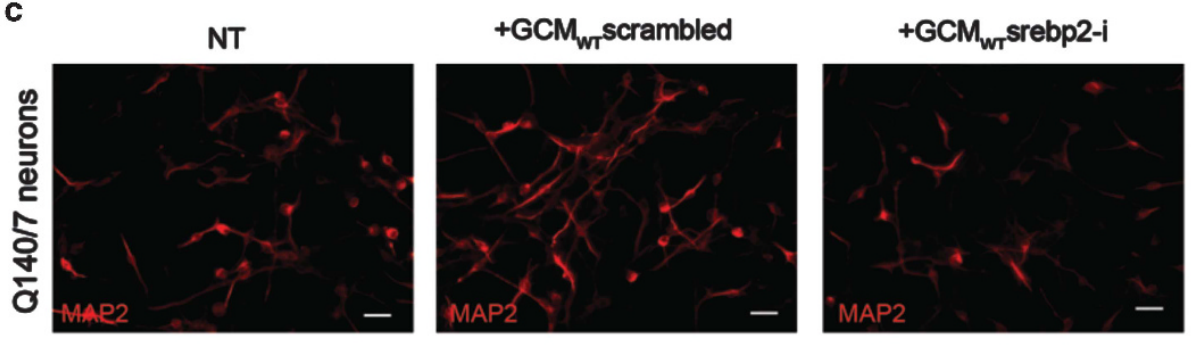

d

e

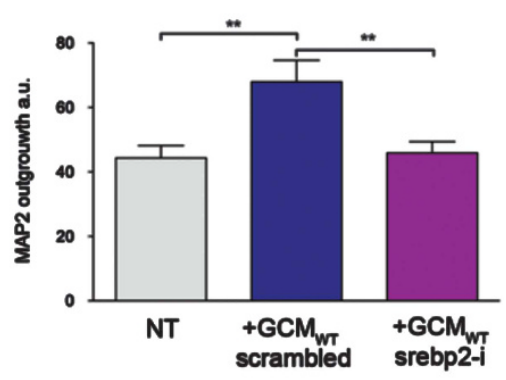

f

GCM R6/2

native PAGE
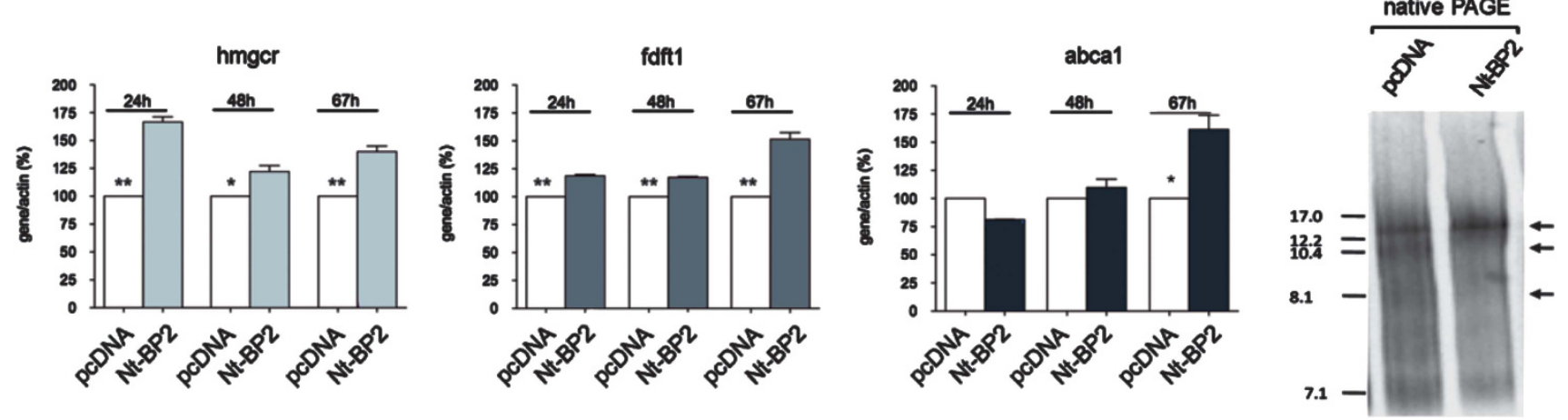

g
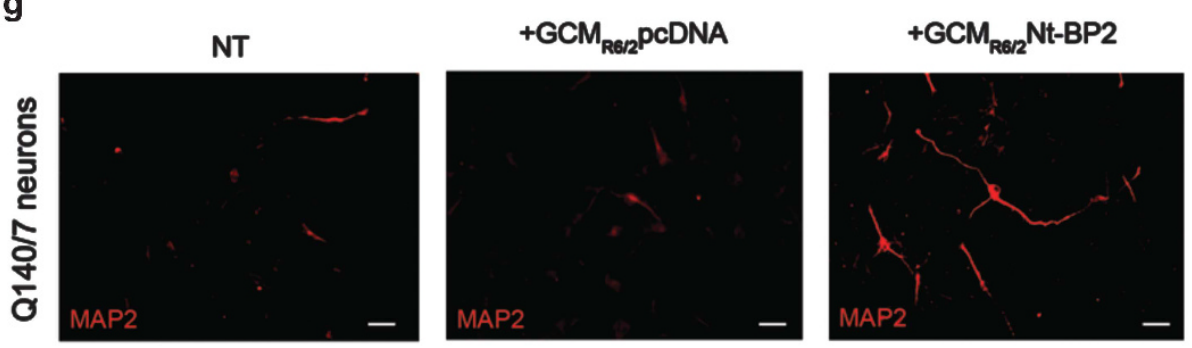

h

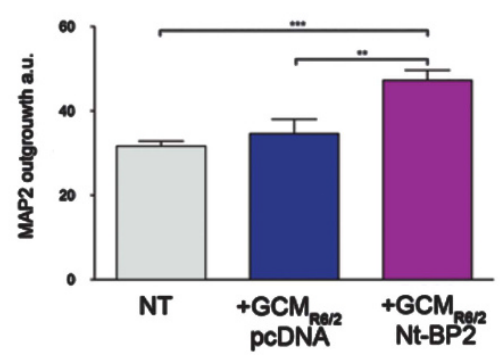

Figure 4 Genetic modulation of SREBP2 in astrocytes influences neurite outgrowth in HD neurons. (a) mRNA levels of srebp2 in wt primary astrocytes after transfection with siRNA against srebp2 (srebp2-i) compared with the same cells transfected with a scramble as control (scrambled) and (b) apoE levels released in the corresponding GCMs. (c) Representative immunofluorescence staining for MAP2 in Q140/7 neurons in glial-free condition (NT), in the presence of $\mathrm{GCM}_{\mathrm{wt}} \mathrm{scrambled}$ or in the presence $\mathrm{GCM}_{\mathrm{wt}} \mathrm{Srebp}-\mathrm{i}$ and (d) relative neurite outgrowth quantification. Scale bars: $25 \mu \mathrm{m}$. (e) mRNA levels of $h m g c r$, fdtt1, and abca1 in primary R6/2 astrocytes overexpressing the N-terminal fragment of SREBP2 (Nt-BP2) or an empty vector (pcDNA) at different time points from the nucleofection. (f) ApoE-containing lipoproteins size in non-denaturing condition in the corresponding GCM from R6/2 astrocytes overexpressing an empty vector (pcDNA) or Nt-BP2. (g) Representative immunofluorescence staining for MAP2 in Q140/7 neurons in glial-free condition (NT), in the presence of $\mathrm{GCM}_{\mathrm{R} / / 2} \mathrm{pCDNA}$ and in the presence $\mathrm{GCM}_{\mathrm{R} / 2} \mathrm{Nt}-\mathrm{BP} 2$, and (h) relative neurite outgrowth quantification. Graphs in $\mathbf{a}$, $\mathbf{b}$, and $\mathbf{e}$ show mean as $\%$ above relative controls \pm S.E.M. Graphs in $\mathbf{d}$ and $\mathbf{h}$ show the mean (a.u.) \pm S.E.M. for one experiment in which 10 fields were analyzed for each condition. Similar results were obtained in other two independent differentiations. Statistics: two-tailed, unpaired $t$ test $(\mathbf{a}, \mathbf{b}$, and $\mathbf{e})$ or one-way ANOVA with Newman-Keuls multiple-comparison post test (d and $\mathbf{h}$ ). ${ }^{\star} P<0.05 ;{ }^{* \star} P<0.01 ;{ }^{* \star} P<0.001$. The samples in $\mathbf{b}$ were run together with $\mathrm{GCM}_{\mathrm{wt}}$ abca-i shown in Figure $3 \mathrm{~b}$. The control sample (GCM $\mathrm{ws}_{\mathrm{w}} \mathrm{s}$. in $\mathbf{b}$ and in Figure $3 \mathbf{b}$ is the same. See Supplementary Figure 9 for full-length pictures of the blots shown in $\mathbf{b}$ 
$\mathrm{HD},{ }^{37}$ highlighting that some electrophysiological features of striatal dysfunction in HD can be secondary to glial disturbances. Here, we demonstrate that HD astrocytes affect neuronal function through their reduced synthesis and secretion of cholesterol bound to apoE lipoproteins.

We found that HD NS-derived neurons displayed neurite outgrowth defects at day 7 of neuronal differentiation when compared with control neurons. Conditioned media from wt astrocytes, but not from HD astrocytes, rescued the neuritic defect, suggesting that cholesterol dysfunction in glial cells may contribute to neuritic morphology defects, thereby compromising neuron function. Although several glialderived factors may contribute to neurite outgrowth in cultured neurons, ${ }^{38}$ we demonstrated a direct link between glial cholesterol and neurite outgrowth in HD neurons. First, we showed that cholesterol administration rescued neuritic defects in HD neurons during early stages of differentiation. This effect was specifically observed with cholesterol concentrations of $7-10 \mu \mathrm{g} / \mathrm{ml}$, suggesting that HD neurons depend on exogenous cholesterol for their activities and that cholesterol concentration is critical for this effect. Notably, this is the same concentration at which cholesterol is found in GCM. ${ }^{11}$ Second, we found that lipoprotein-depleted GCM from wt astrocytes did not support neurite outgrowth and synaptic properties in HD neurons, pointing out a role of lipoproteins in neuronal function. Third, media conditioned by primary astrocytes from apoE ${ }^{-1-}$ mice did not improve neuronal properties in HD neurons, despite the presence of higher levels of apoD, supporting the unique role of cholesterol bound to apoE lipoproteins present in GCM. Finally, abca1 silencing in wt astrocytes negatively influenced neurite outgrowth in HD neurons, whereas its overexpression in HD astrocytes had the opposite effect. This is in agreement with the critical role of ABCA1 in brain cholesterol metabolism and neuronal function. ${ }^{39}$ The evidence that Irp1 mRNA levels are similar in wt and R6/2 brains also confirms that HD astrocytes are the mediators of cholesterol dysfunction in HD. Modulation of cholesterol efflux might be an alternative strategy for targeting cholesterol-dependent neuronal defects in HD. Agonists of liver-X receptors induced transcription of $A B C A 1$ and apoE, reduced amyloid- $\beta$ levels and improved cognition in Alzheimer's disease, ${ }^{40}$ and may reduce neuroinflammation. ${ }^{41}$ Moreover, an liver-X receptor agonist partially rescued HTT knockdown phenotypes in Zebrafish. ${ }^{42}$ However, further studies are needed to evaluate the potential of these drugs in HD and to develop more brain-specific liver-X receptor agonists.

Our findings suggest a prominent role of glial SREBP2 in neuronal function. SREBP2 is expressed in glial cells and its activity likely controls the synthesis of lipids involved in various glia-neuron interactions, thereby affecting a range of neuronal functions. ${ }^{43-45}$ SREBP1 and SREBP2 activities are reduced in the presence of mutant HTT, both in vitro and in vivo. ${ }^{7}$ Although the details of the underlying molecular mechanism are unknown, mutant HTT likely interferes with the Golgi-tonucleus translocation of the active SREBP. ${ }^{7}$ The effects on neurite outgrowth in HD neurons following the silencing or overexpression of active SREBP2 in astrocytes demonstrate a link between cholesterol synthesis in astrocytes and subsequent efflux likely under the regulating effect of oxysterols, such as $24 \mathrm{OHC}{ }^{46}$ Reduced levels of $24 \mathrm{OHC}$ in $\mathrm{HD}^{8-10}$ may therefore exacerbate cholesterol unbalance between astrocytes and neurons.

In the absence of cell death, HD animal models exhibit abnormalities in synaptic communication ${ }^{47,48}$ and neuronal dysfunction precedes cell death by many years in HD humans. ${ }^{49}$ Similarly, cholesterol biosynthesis disruption in HD animal models and patients occurs before the onset of motor defect.9,10,18,50 Additionally, synaptosomes carry suboptimal levels of sterols in early R6/2 model. ${ }^{8}$ Here, we showed that a non-cell autonomous cholesterol-handling defect in astrocytes affects cholesterol shuttling between astrocytes and neurons, which has a reversible detrimental effect on synaptic-related parameters in HD neurons.

How does reduced glial cholesterol contribute to neuronal dysfunction in HD? Cholesterol promotes different aspects of synapse development and maintenance, including dendrite differentiation, synaptic vesicle formation and release, and receptor clustering and stability. ${ }^{12-53}$ Of note, proteome-wide mapping of cholesterol-interacting proteins in mammalian cells reveals that most of these proteins are linked to neurological disorders. ${ }^{54}$ Neurons produce cholesterol less efficiently than glial cells ${ }^{55}$ and the appearance of most synapses in the developing brain is temporally and spatially coincident with astrocyte development, suggesting that synapse formation may depend on astrocytes-derived cholesterol. ${ }^{56}$ Changes in cholesterol pathways and in apoE expression have been also associated to NMDA-mediated excitotoxicity. ${ }^{57-59}$ Of note, $24 \mathrm{OHC}$ is a potent and specific modulator of NMDA receptors. ${ }^{60}$ Although cholesterol homeostasis has been reported to contribute to enhanced excitotoxicity in $\mathrm{HD},{ }^{61}$ however, further studies are needed to address this relationship.

In conclusion, our results indicate that HD astrocytes are responsible for cholesterol dysfunction in the HD brain, by supplying less cholesterol to the surrounding neurons. Glial SREBP2 might be a candidate target for in vivo approaches aiming to explore the contribution of glial cholesterol dysfunction in HD. Its enhancement may contribute to attenuating neurite degeneration and synaptic defects in HD neurons.

\section{Materials and Methods}

Neural stem cell lines and neuronal differentiation. The NS cell lines employed in this study were grown and differentiated towards neurons for 7 days, as previously described. ${ }^{17}$

Primary cultures of neurons. We used primary neurons to measure synaptic properties because NS-derived neurons did not reach complete functional maturation in vitro (data not shown). Primary cultures of striatal neurons were generated from transgenic R6/2 mice that overexpress exon 1 of the human HTT gene containing $150 \mathrm{CAG}$ repeats. At embryonic day $(\mathrm{E})$ 16.5, tails from each embryo were used to extract genomic DNA and to perform PCR for genotype determination. Next, the brain was dissected and tissue was placed into ice-cold HIBERNATE MEDIA (Life Technologies Italia, Monza, Italy). Cells were obtained first through an enzymatic disaggregation with $0.5 \mathrm{mg} / \mathrm{ml}$ papain (Worthington biochemical Corporation, Lakewood, NJ, USA) for $15 \mathrm{~min}$ at $37^{\circ} \mathrm{C}$, and then by mechanical dissociation. The dissociated cells were resuspended in DMEM (Invitrogen, Life Tecnhologies Italia) supplemented with $10 \%$ fetal bovine serum, $0.5 \mathrm{mM} \mathrm{L-glutamine} \mathrm{(Invitrogen),} \mathrm{and} 100 \mathrm{U} / \mathrm{ml}$ of penicillin-streptomycin (Invitrogen). They were then seeded at a density of 400000 cells per well in 12-well plates or 1000000 cells per well in 6-well plates. After 1 div, the medium was totally replaced with DMEM/F12 supplemented with B27 $1 \times$ (Life Technologies), $0.5 \mathrm{mM}$ 
a

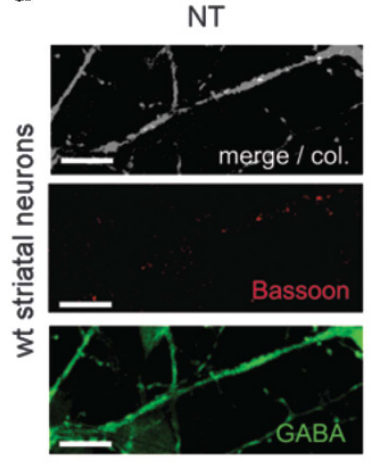

b

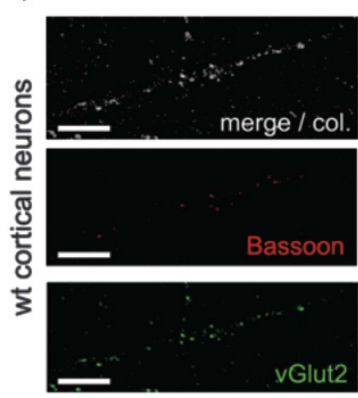

NT

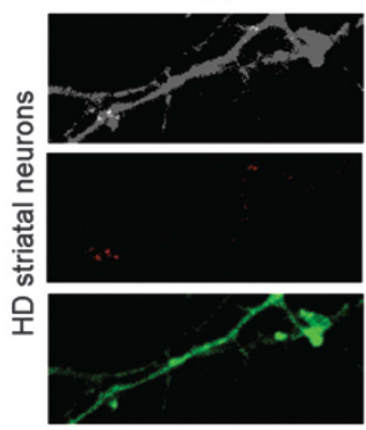

NT

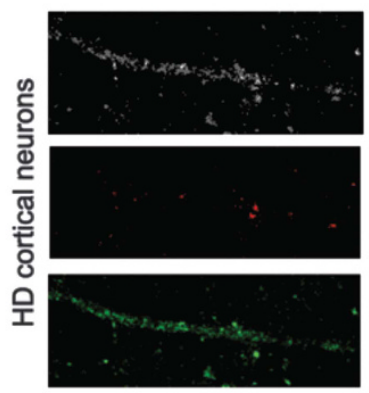

+ GCM wt

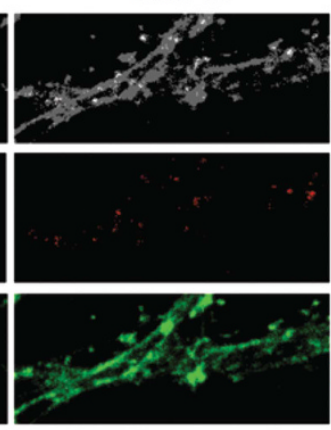

+ GCM wt
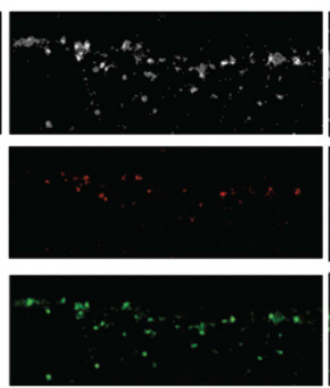

+ GCM HD

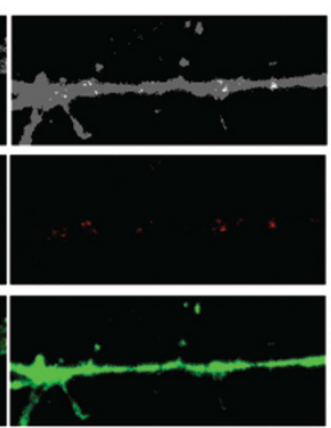

+ GCM HD
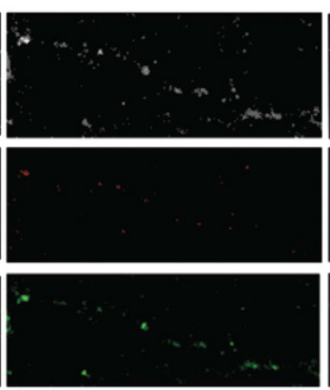

+ GCM wt delip

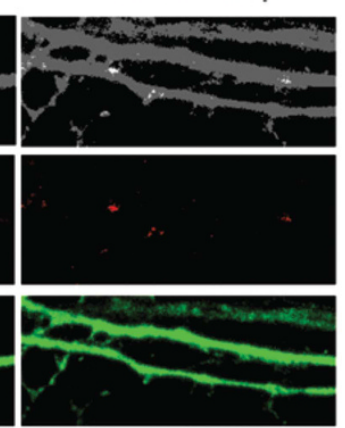

+ GCM wt delip

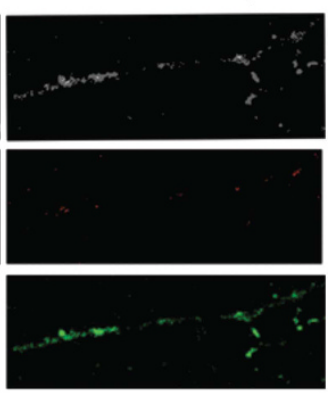

c
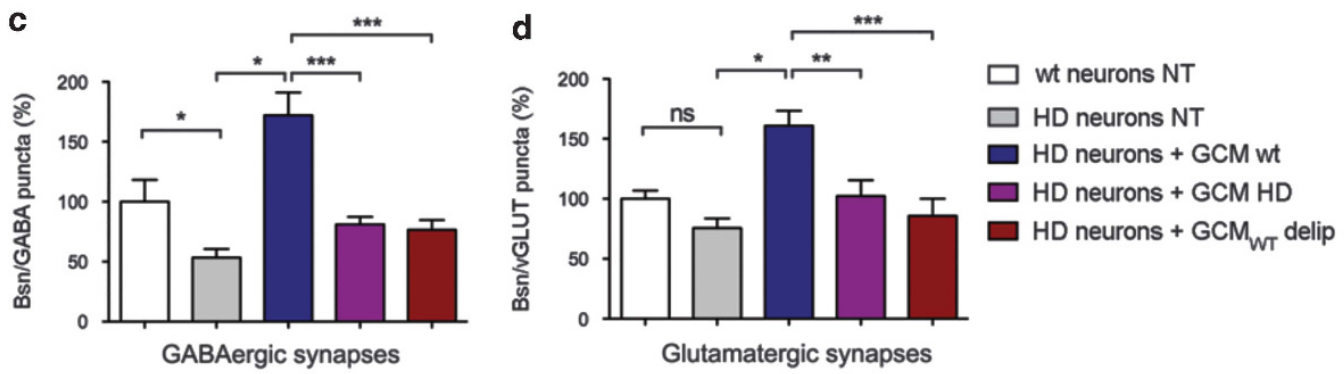

e

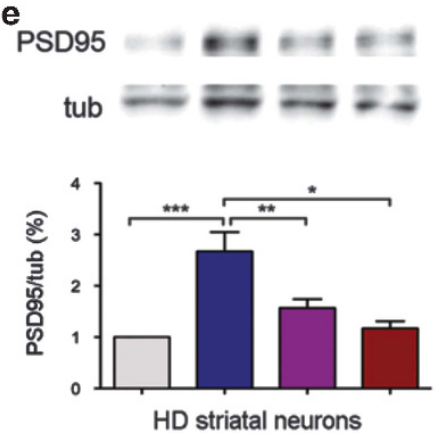

f
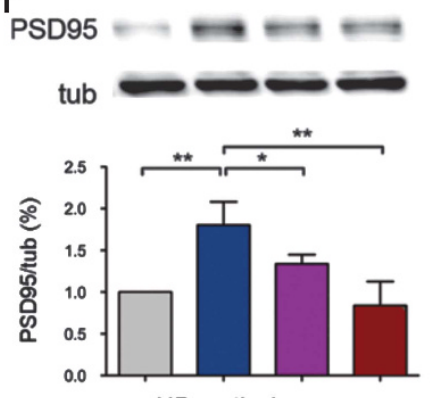

HD neurons NT

$\square$ HD neurons + GCM wt

$\square$ HD neurons + GCM HD

$\square$ HD neurons + $\mathrm{GCM}_{\mathrm{WT}}$ delip

HD cortical neurons

Figure 5 GCM HD does not promote synaptic properties in HD neurons. (a and $\mathbf{b}$ ) Fluorescence images of primary cortical and striatal neurons under glial-free conditions (NT) or in the presence of GCM wt, GCM R6/2, or GCM delip, double-immunostained with Bassoon (red, middle panels) and either VGLUT-2 or GABA (green, bottom panels), along with the relative co-localization (merge; col.). (c and d) Quantification of puncta that are double-positive for Bassoon and either VGLUT-2 or GABA to identify glutamatergic and GABAergic bona-fide synapses, respectively. Scale bars: $5 \mu \mathrm{m}$. (e and f) Western blot analysis for PSD95 in protein lysates from primary R6/2 (HD) striatal and cortical neurons under glia-free conditions (NT) or in the presence of GCM wt, GCM R6/2, or GCM delip. (c and d) Graphs show the mean \% above relative controls \pm S.E.M. from 10 fields each for three independent experiments. Statistics: one-way ANOVA with Newman-Keuls multiple-comparison post test. ${ }^{*} P<0.05 ;{ }^{* \star} P<0.01$; ${ }^{\star \star \star} P<0.001$. See Supplementary Figure 9 for full-length pictures of the blots shown in $e$ and $f$

L-glutamine (Invitrogen), and $100 \mathrm{U} / \mathrm{ml}$ of penicillin-streptomycin (Invitrogen). Cells were cultured for 11 div with multiple medium changes.

Glial-conditioned medium preparation. NS-derived astrocytes were obtained by plating NS cells on uncoated $25-\mathrm{cm}^{2}$ flasks, and exposing them to
Glasgow Minimum Essential Medium (Celbio, Euroclone S.p.a., Milan, Italy) in the presence of $10 \%$ fetal bovine serum, $0.5 \mathrm{mM}$ L-glutamine (Invitrogen), and $100 \mathrm{U} / \mathrm{ml}$ of penicillin-streptomycin (Invitrogen) for 14 days, with the medium changed every 3 days. Primary astrocytes were generated from postnatal day 1 pups as previously described. ${ }^{8}$ Astrocytes were seeded in six-well plates 
(1600 000/well) and, $24 \mathrm{~h}$ later, were exposed to serum-free DMEM/F12 (Invitrogen) for $6-8 \mathrm{~h}$. Conditioned medium was collected, centrifuged at $1000 \times \mathrm{g}$ for $4 \mathrm{~min}$, and then concentrated 10-fold using Amicon Ultra-4 filter devices (Millipore, Billerica, MA, USA). Lipoprotein-depleted GCM was prepared by mixing $5 \mathrm{ml}$ of GCM with

a

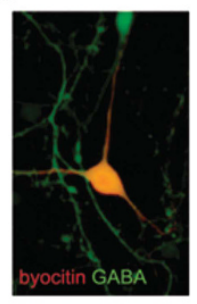

b

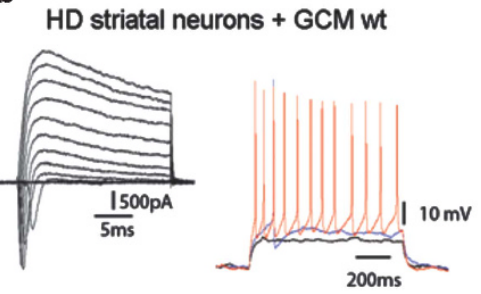

$50 \mathrm{mg}$ of colloidal silica that avidly binds lipoproteins (Cab-o-sil, Sigma-Aldrich, St Louis, MO, USA). After 30 min of mixing, the Cab-o-sil (which contained bound lipoproteins) was removed from the medium by centrifugation for 5 min at $1000 \times g$. Immunoblotting showed that the Cab-o-sil-treated GCM contained no detectable

\section{C}

HD striatal neurons + GCM HD

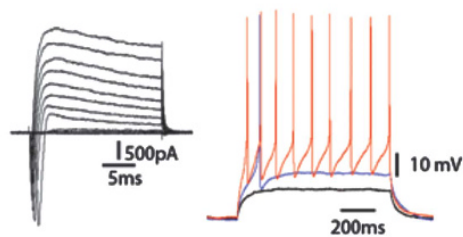

e

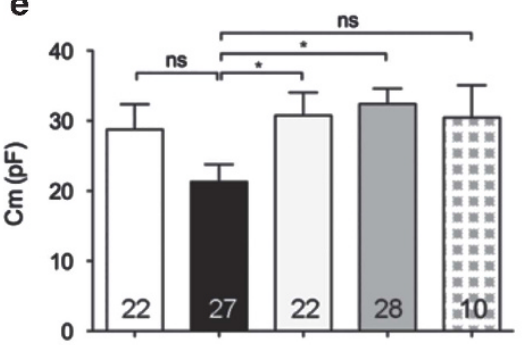

$\square$ wt NT

HD NT

HD + GCM wt HD + GCM HD HD + GCM HD + cho

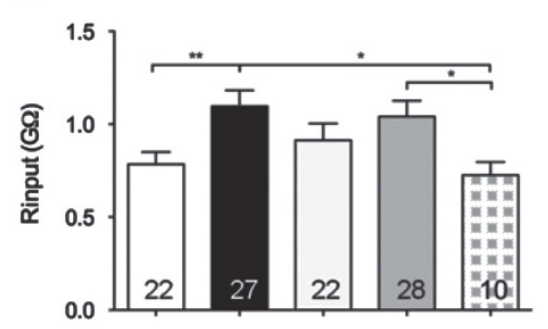

f

wt striatal neurons NT

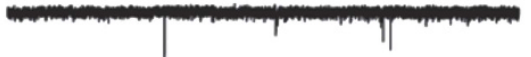

HD striatal neurons NT

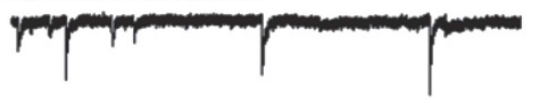

HD striatal neurons + GCM wt

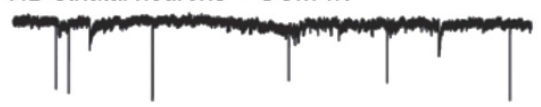

HD striatal neurons + GCM HD

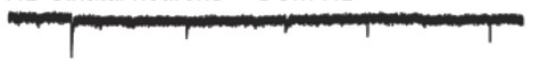

HD striatal neurons + GCM HD + chol

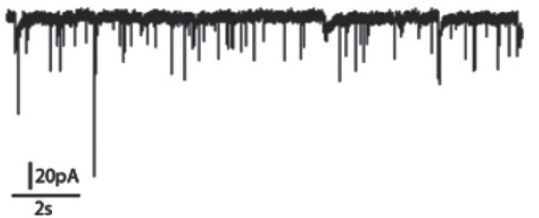

g

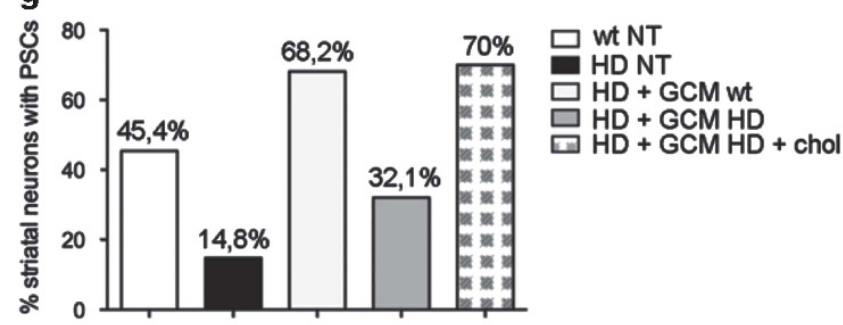

h

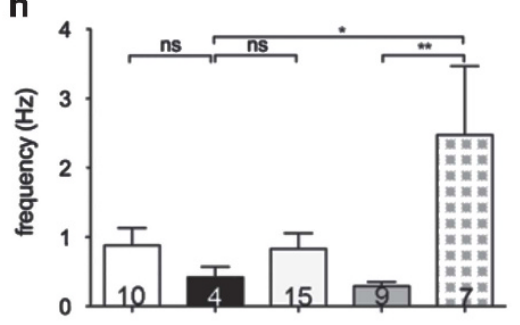

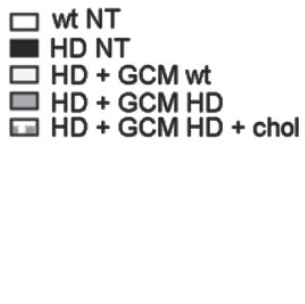

i

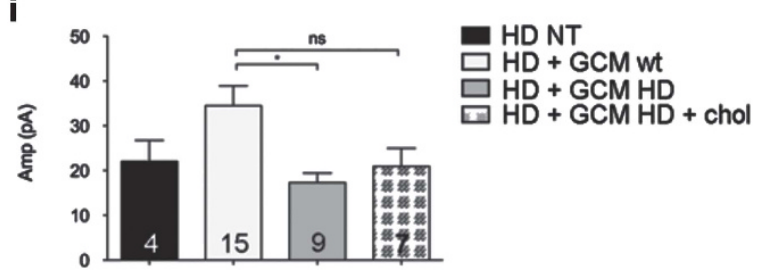

Figure 6 GCM wt, but not GCM HD, stimulates synaptic activity in HD neurons. (a) Representative fluorescent image of a recorded primary striatal neuron immunostained for biocytin and GABA. (b and c) Family of fast inward (negative) and outward (positive) currents, reflecting the presence of sodium ( $\mathrm{Na}+$ ) and potassium ( $\mathrm{K}+$ ) channels, respectively, recorded in response to depolarizing $10 \mathrm{mV}$ voltage step (from -90 to $+50 \mathrm{mV}$ ) and sample traces of repetitive action potentials in primary R6/2 (HD) striatal neurons recorded in response to increasing current step injection from a holding potential of $-70 \mathrm{mV}$ in the presence of GCM wt or GCM HD. (d and e) Histograms showing the input resistance (Rin) (d) and the membrane capacitance $(\mathrm{Cm})(\mathbf{e})$ in primary wt and $\mathrm{HD}$ striatal neurons under different medium conditions. (f) Sample current traces of spontaneous synaptic activity recorded in voltage-clamp at $-70 \mathrm{mV}$. (g-i) Histograms showing the \% of neurons able to produce spontaneous PSCs $(\mathbf{g})$, the mean frequency (h), and the mean amplitude (i) of synaptic events. Data in $\mathbf{d}, \mathbf{e}, \mathbf{g}, \mathbf{h}$, and $\mathbf{i}$ are shown as mean \pm S.E.M. Numbers inside the columns represent the number of recorded cells (d and $\mathbf{e})$ or recorded cells showing PSCs (h). $P$ values were determined by non-parametric Mann-Whitney test. ${ }^{\star} P<0.05 ;{ }^{\star \star} P<0.01 ;{ }^{\star \star \star} P<0.001$; ns $=$ not statistically significant 
apoE. Each stock of GCMs used for subsequent experiments was first checked for apoE levels.

Medium apoE analysis. Equal volumes of concentrated media were resolved on Criterion TGX Stain-Free Gels (Bio-Rad, Hercules, CA, USA) with SDS. These stain-free gels contain proprietary trihalo compounds that react with tryptophan residues, producing fluorescent light through an ultraviolet light-induced reaction. Stain-free imaging of the gels and membranes, corresponding to proteins in the samples, was used as loading control and for normalization. ${ }^{62}$ Proteins were transferred to a nitrocellulose membrane using a Trans-Blot Turbo system (BioRad). They were then probed with a polyclonal goat anti-apoE antibody $(1: 800$; AB947, Millipore), washed, and probed with HRP-conjugated horse anti-goat IgG (1:3000; Bio-Rad). Bands were visualized with enhanced chemoluminescence (Pierce, Thermo Scientific, Rockford, IL, USA) and imaged with ChemiDoc MP Imaging System (Bio-Rad).

Nondenaturing gradient gel electrophoresis. GCM samples were mixed 1:1 with native sample buffer (Bio-Rad) and electrophoresed on Criterion TGX Stain-Free 4-15\% Gels (Bio-Rad). Proteins were transferred to a nitrocellulose membrane and probed with apoE antibody as described above. Proteins with known hydrated diameters were used as size standards (GE Healthcare, Buckinghamshire, UK).

ApoE ELISA. For in vitro quantitative determination of mouse apoE concentration in GCMs, we used the Mouse apoE ELISA Kit (MyBioSource, San Diego, CA, USA) following the manufacturer's procedures. Only fresh media were used for the analyses.

Measurement of total cholesterol. Total cholesterol content was evaluated in 10-fold concentrated GCMs. A colorimetric assay was performed using the Cholesterol Kit (Amplex Red Cholesterol Assay Kit, Invitrogen) following the manufacturer's instructions.

Immunofluorescence. Cells were fixed in 4\% paraformaldehyde for $15 \mathrm{~min}$ at room temperature, and washed three times with PBS. The cells were permeabilized with Triton $0.5 \%$ (Sigma) and blocked with $5 \%$ fetal bovine serum (Euroclone S.p.a.) for $1 \mathrm{~h}$ at room temperature. Cells were then incubated overnight at $4{ }^{\circ} \mathrm{C}$ with one of the following primary antibodies: mouse anti-MAP2 (1:500; Becton Dicknson, Franklin Lakes, NJ, USA), rabbit anti-VGLUT2 (1:500; Synaptic Systems GmbH, Goettinger, Germany), rabbit anti-GABA (1:500; Sigma-Aldrich), mouse anti-GFAP (1: 500; Becton Dickinson), and rabbit anti-S100 $\beta$ (1:200; Sigma-Aldrich). Next, the cells were washed three times with PBS, followed by a 1-h incubation at room temperature with the appropriate secondary antibodies conjugated to 488 or 568 Alexa-fluorophores (Molecular Probes, Life Technologies). Finally, the cells were washed three times with PBS, and the nuclei were stained with Hoechst 33258 ( $5 \mathrm{mg} / \mathrm{ml}$; Molecular Probes, Life Technologies). Images were acquired with a Leica DMI 6000B microscope (Leica microsystems, Wetzlar, Germany) with $\times 10$ and $\times 20$ objectives, using LAS-AF imaging software (Leica microsystems). Adobe Photoshop CS3 (Adobe Systems Incorporated, San Jose, CA, USA) was used to increase the image quality by applying the same values of luminosity and contrast to all images being compared.

Neurite outgrowth quantification. Total neurite length was estimated using the ImageJ plugin Neurite Tracer $^{20}$ in combination with a modified macro for counting neuronal nuclei that was developed in the lab. The analysis was performed blind to the experimental conditions. In the main figures, the data are represented as the mean (a.u.) \pm S.E.M. of one experiment in which 10 fields were analyzed for each condition. Graphs showing neurite outgrowth quantification expressed as \% above the relative controls, as mean of all the independent experiments performed in this work have been showed in Supplementary Figure 8.

Flow cytometry. Detached cells were fixed in $0.1 \%$ paraformaldehyde, permeabilized for $15 \mathrm{~min}$ in a $0.2 \%$ Tween 20 solution, and resuspended in fetal bovine serum. We then added a solution containing primary antibodies (anti-MAP2, $1: 500$, Becton Dickinson; anti-GFAP, $1: 500$, Becton Dickinson; anti-S100 $\beta, 1: 200$, Sigma-Aldrich). After washing, appropriate Alexa488- and 647-conjugated secondary antibodies were used $(1: 1000)$. Primary isotypic antibodies were used as controls. Analyses were performed using a FACS Canto II analyzer (BD
Biosciences, San Jose, CA, USA) and BD FACSDiva v6.1.3 software. For each condition, 10000 cells were analyzed.

Cell proliferation assays. On day 3 of neuronal differentiation, NS cells were plated on laminin-coated $\left(3 \mu \mathrm{g} / \mathrm{ml}\right.$ for $3-5 \mathrm{~h}$ at $\left.37^{\circ} \mathrm{C}\right)$ 96-well microplates, at $30 \times 10^{3}$ cells/well. The CyQuant NF Cell Proliferation Assay Kit (Molecular Probes, Invitrogen) was used to assay cell viability after treatment of the cells for $24,48,72$, and $96 \mathrm{~h}$ with different doses of cholesterol, following the manufacturer's procedures.

Nucleofection. The Amaxa Cell Line Optimization Nucleofector Kit (Lonza, Basel, Switzerland) was used to modulate gene expressions in different cell lines. Electroporation was performed using pre-set programs: T-020 for the nucleofection of primary astrocytes, and A-033 for NS-derived neurons. The following constructs were used: specific siRNA for abca, srebp2 and Irp1 (Santa Cruz Biotechnology, Inc., Santa Cruz, CA, USA), plasmid overexpressing ABCA1 (gift by S. Calandra, University of Modena) and plasmid overexpressing the active fragment of SREBP2 (as described previously ${ }^{6}$ ).

RNA isolation, retrotranscription, and real-time PCR for gene expression. Total RNA from cell cultures was isolated with TRIZOL Reagent (Invitrogen). Total RNA $(0.25-0.5 \mu \mathrm{g})$ was reverse-transcribed to single-stranded cDNA using the iScript cDNA synthesis kit (Bio-Rad). For each reverse-transcribed product, three real-time PCR analyses were performed in duplicate for each of the analyzed genes. An iCycler Thermal Cycler with a Multicolor Real-time PCR Detection System (Bio-Rad) was used to evaluate gene expressions. All reactions were performed in a total volume of $15 \mu$, containing $60 \mathrm{ng} \mathrm{cDNA}, 50 \mathrm{mM} \mathrm{KCl}$, $20 \mathrm{mM}$ Tris- $\mathrm{HCl}$ ( $\mathrm{pH} 8.4$ ), $0.2 \mathrm{mM}$ dNTPs, $25 \mathrm{U} / \mathrm{ml}$ iTaq DNA polymerase, $3 \mathrm{mM}$ $\mathrm{MgCl}_{2}$, SYBR Green I, 10 nM fluorescein, stabilizers (iQ EVA Green Supermix; Bio$\mathrm{Rad})$, and $0.2 \mu \mathrm{M}$ forward and reverse primers. Amplification cycles consisted of an initial denaturing cycle at $95^{\circ} \mathrm{C}$ for $3 \mathrm{~min}$, followed by 45 cycles of $30 \mathrm{~s}$ at $95^{\circ} \mathrm{C}$, $30 \mathrm{~s}$ at $60^{\circ} \mathrm{C}$ (for all analyzed genes), and $30 \mathrm{~s}$ at $72^{\circ} \mathrm{C}$. Fluorescence was quantified during the annealing step, primer specificity and product formation were confirmed by melting curve analysis $\left(55-94^{\circ} \mathrm{C}\right)$, and the amounts of target gene mRNA were normalized to $\beta$-actin as reference gene and by using the calibration curve method. The following primer sequences were used: $\mathrm{Hmgcr}$ fwd, GGAGCATAGGCGGCTACA; Hmgcr rev, ACCACCCACGGTTCCTATCT; Fdft fwd, ACTCAGCAGCAGCTTGAAGACC; Fdft rev, TGTCATCCTCCACTGTATCCAG; Abca1 fwd, GTCAGCTGTTACTGGAAGTGG; Abca1 rev, CGCCGGGAGTTGGATA ACGG; Irp1 fwd, AGGCCACCTCTGCAGCTGT; Irp1 rev, GCTGCGGATCTCGTTG TCATC; Actin fwd, AGTGTGACGTTGACATCCGTA; Actin rev, GCCAGAGCAGTA ATCTCCTTCT; Srebp2 fwd, GCCTCTCCTTTAACCCCTTG; and Srebp2 rev, CCAGTCAAACCAGCCCCCAG.

Quantification of bona fide synapses. Following the above-described procedures, we performed standard immunofluorescence on primary neuronal cultures with a mouse anti-Bassoon antibody (1:400; Stressgen Biotechnologies, Collegeville, PA, USA) and with the specific neuronal markers v-GLUT2 and GABA. Confocal images were acquired with a Zeiss LSM 510 laser-scanning confocal microscope with AlM4.2 software (Zeiss, Oberkochen, Germany). The number of co-localizations was quantified using the 'Red and Green Puncta Colocalization' macro under the ImageJ analysis software platform (U.S. National Institutes of Health, Bethesda, MD, USA).

Western blot analysis. Cells were lysed by adding $50 \mu \mathrm{l}$ of RIPA buffer supplemented with PMSF (1:250; Sigma-Aldrich) and protease inhibitors (1:100; Sigma-Aldrich). After centrifugation at $10000 \times g$ for $5 \mathrm{~min}$, the supernatant was collected and the protein concentration was determined by BCA assay (Thermo Scientific). Nuclear extracts to evaluate the active form of SREBP2 were prepared as previously described. ${ }^{7}$ Proteins were separated by SDS-polyacrylamide gel electrophoresis and then transferred to a nitrocellulose membrane using a TransBlot Turbo System (Bio-Rad). The membranes were next probed with mouse antiPSD95 (1:1000; Synaptic Systems), rabbit anti-ABCA1 (1:500; gift from M. Hayden), goat anti-apoE (1:800; AB947, Millipore), mouse anti- $\alpha$-tubulin (1:5000; Sigma-Aldrich), rabbit anti-SREBP2 (1:200; Abcam, Cambridge, MA, USA) followed by washing, and probing with horseradish peroxidase-conjugated secondary antibodies (1:3000; Bio-Rad). Bands were visualized with enhanced chemoluminescence (Pierce) and imaged with the ChemiDoc MP Imaging System (Bio-Rad). 
Electrophysiological analyses. We recorded 157 primary striatal neurons, of which $127(80.9 \%)$ showed $\mathrm{Na}^{+}$currents with amplitude larger than $500 \mathrm{pA}$, suggesting that most of the recorded cells were functionally mature and potentially able to generate action potentials. Cells were visualized using an inverted microscope (Eclipse TE200, Nikon, Tokyo, Japan) equipped with both $\times 10$ and $\times 40$ objectives. During recording, primary neurons were maintained at room temperature in a bath solution containing $140 \mathrm{mM} \mathrm{NaCl}, 2 \mathrm{mM} \mathrm{CaCl}_{2}, 1 \mathrm{mM} \mathrm{MgCl}_{2}, 3 \mathrm{mM} \mathrm{KCl}$, $10 \mathrm{mM}$ glucose, and $10 \mathrm{mM}$ HEPES, adjusted to $\mathrm{pH} 7.4$ with $\mathrm{NaOH}$. Patch pipettes with a resistance of 3-5 M $\Omega$ were fabricated from thick-walled borosilicate glass capillaries using a Sutter P-97 horizontal puller (Sutter Instruments, Novato, CA, USA), and filled with the following intracellular solution: $130 \mathrm{mM}$ potassium gluconate, $4 \mathrm{mM} \mathrm{NaCl}, 10 \mathrm{mM}$ HEPES, $1 \mathrm{mM}$ EGTA, $2 \mathrm{mM} \mathrm{MgCl}$, $5 \mathrm{mM} \mathrm{CP}$, adjusted to $\mathrm{pH} 7.3$ with $\mathrm{KOH}$.

Recordings were obtained in the whole-cell configuration, both in voltage- and current-clamp mode, using an Axoclamp 200B amplifier (Molecular Devices) and a Digidata converter AD/DA (Molecular Devices). Signals were low-pass filtered at $10 \mathrm{kHz}$, and acquired at $10-50 \mathrm{kHz}$ with Clampex software (Molecular Devices, Sunnyvale, CA, USA). To appreciate the morphology, cells were filled either with biocytin (3 mg/ml) or Alexa Fluor 488 (Molecular Probes, Invitrogen), routinely added to the intracellular solution. In voltage-clamp configuration, passive membrane properties were measured immediately after the break-in. The input resistance (Rin) was calculated from the current trace at the end (mean of the last $5 \mathrm{~ms}$ ) of the 180 $\mathrm{ms}$-long voltage step from $-70 \mathrm{mV}$ to $-80 \mathrm{mV}$. Using the same protocol, the membrane capacitance $(\mathrm{Cm})$ was measured by subtracting the time-integral of the steady-state current from the total time-integral of the current transient developing during the $10 \mathrm{mV}$ voltage step and dividing by the same voltage step. Spontaneous synaptic activity was recorded in the voltage-clamp configuration at $-70 \mathrm{mV}$. Negative deflections of current trace were acknowledged as spontaneous synaptic events, and the peak amplitude was evaluated as the mean over $300 \mu \mathrm{s}$ around a local minimum. Frequency was calculated as the ratio between the number of synaptic events and the time window of $30-60 \mathrm{~s}$, and the mean amplitude was calculated by selecting a sufficient number $(>30)$ of well-isolated events. In currentclamp configuration an appropriate holding current was applied to maintain the membrane potential at $-70 \mathrm{mV}$ and 1-s-long positive current steps of increasing amplitude were injected to investigate the ability to generate an action potential or a repetitive firing. All chemicals were from Sigma-Aldrich.

Statistical analysis. No statistical methods were used to predetermine sample sizes, but our sample sizes were similar to those reported in literature. Data were expressed as mean \pm S.E.M. Statistical analysis was performed by two-tail Student's t-test or one-way ANOVA with Newman-Keuls Multiple Comparison posthoc test. The Mann-Whitney test was used to assess the input resistance, membrane capacitance, and post-synaptic current frequency and amplitude-all variables for which normality of the data could not be assumed. Normality of the data was assessed using the Shapiro-Wilk test. Outliers were identified with Grubbs' test, and were excluded from the analyses. Differences for which $P$ was $<0.05$ were considered to be significant.

\section{Conflict of Interest}

The authors declare no conflict of interest.

Acknowledgements. This work was supported by the Cure Huntington Disease Initiative (CHDI, USA, no. A-5086), the Telethon Foundation (Italy, no. GGP12122), and, partially, by Neuromics (Integrated European -omics research project for diagnosis and therapy in rare neuromuscular and neurodegenerative diseases no. 305121) to EC; by Fondazione Cariplo (Italy, no. 2008/2406) to MV; and by PRIN (Ministero dell'Istruzione, della Università $e$ della Ricerca, Italy, no. 2010JMMZLY) to GB. We thank Sebastiano Calandra (University of Modena) for providing us with the ABCA1 plasmid, Alessandro leraci (University of Milan) for technical support in the initial generation of primary neurons, and Giulia Chiesa and Marco Busnelli (University of Milan) for providing us with $\mathrm{apo} \mathrm{E}^{-1-}$ pups.

\section{Author contributions}

$\mathrm{EC}, \mathrm{MV}$, and MM developed the study, conceived the experimental plans, and analyzed the data. MM, MV, and EDP performed most of the biological, biochemical, and molecular experiments. $\mathrm{ECe}$ and $\mathrm{GB}$ were involved in all of the electrophysiological experiments and the interpretation of the results. $\mathrm{CZ}$ provided suggestions for some biological experiments. MV, MM, and EC interpreted the data and wrote the manuscript. All of the authors read and edited the manuscript. EC supervised the entire work, directed the strategies, and gave final approval of the version to be published.

1. Zuccato C, Valenza M, Cattaneo E. Molecular mechanisms and potential therapeutical targets in Huntington's disease. Physiol Rev 2010; 90: 905-981.

2. Dietschy JM, Turley SD. Thematic review series: brain Lipids. Cholesterol metabolism in the central nervous system during early development and in the mature animal. $J$ Lipid Res 2004; 45: 1375-1397.

3. Porter FD, Herman GE. Malformation syndromes caused by disorders of cholesterol synthesis. J Lipid Res 2011; 52: 6-34.

4. Suzuki R, Ferris HA, Chee MJ, Maratos-Flier E, Kahn CR. Reduction of the cholesterol sensor SCAP in the brains of mice causes impaired synaptic transmission and altered cognitive function. PLOS Biol 2013; 11: e1001532.

5. Valenza M, Cattaneo E. Emerging roles for cholesterol in Huntington's disease. Trends Neurosci 2011; 34: 474-486.

6. Karasinska JM, Hayden MR. Cholesterol metabolism in Huntington disease. Nat Rev Neurol 2011; 7: 561-572.

7. Valenza M, Rigamonti D, Goffredo D, Zuccato C, Fenu S, Jamot L et al. Dysfunction of the cholesterol biosynthetic pathway in Huntington's disease. J Neurosci 2005; 25: 9932-9939.

8. Valenza M, Leoni V, Karasinska JM, Petricca L, Fan J, Carroll J et al. Cholesterol defect is marked across multiple rodent models of Huntington's disease and is manifest in astrocytes. J Neurosci 2010; 30: 10844-10850.

9. Leoni V, Mariotti C, Tabrizi SJ, Valenza M, Wild EJ, Henley SM et al. Plasma 24Shydroxycholesterol and caudate MRI in pre-manifest and early Huntington's disease. Brain 2008; 131: 2851-2859.

10. Leoni V, Long JD, Mills JA, Di Donato S, Paulsen JS. PREDICT-HD study group Plasma 24Shydroxycholesterol correlation with markers of Huntington disease progression. Neurobiol Dis 2013; 55: 37-43.

11. Mauch DH, Nagler K, Schumacher S, Goritz C, Muller EC, Otto A et al. CNS synaptogenesis promoted by glia-derived cholesterol. Science 2001; 294: 1354-1357.

12. Pfrieger FW, Ungerer N. Cholesterol metabolism in neurons and astrocytes. Prog Lipid Res 2011; 50: 357-371.

13. Hebb MO, Denovan-Wright EM, Robertson HA. Expression of the Huntington's disease gene is regulated in astrocytes in the arcuate nucleus of the hypothalamus of postpartum rats. FASEB J 1999; 13: 1099-1106.

14. Shin JY, Fang ZH, Yu ZX, Wang CE, Li SH, Li XJ. Expression of mutant huntingtin in glial cells contributes to neuronal excitotoxicity. J Cell Biol 2005; 171: 1001-1012.

15. Bradford J, Shin JY, Roberts M, Wang CE, Li XJ, Li S. Expression of mutant huntingtin in mouse brain astrocytes causes age-dependent neurological symptoms. Proc Natl Acad Sci USA 2009; 106: 22480-22485.

16. Bradford J, Shin JY, Roberts M, Wang CE, Sheng G, Li S et al. Mutant huntingtin in glial cells exacerbates neurological symptoms of Huntington disease mice. J Biol Chem 2010; 285: 10653-10661.

17. Conforti P, Camnasio S, Mutti C, Valenza M, Thompson M, Fossale E et al. Lack of huntingtin promotes neural stem cells differentiation into glial cells while neurons expressing huntingtin with expanded polyglutamine tracts undergo cell death. Neurobiol Dis 2013; 50: 160-170.

18. Valenza M, Carroll JB, Leoni V, Bertram LN, Bjorkhem I, Singaraja RR et al. Cholesterol biosynthesis pathway is disturbed in YAC128 mice and is modulated by huntingtin mutation. Hum Mol Genet 2007; 16: 2187-2198.

19. Spiliotopoulos D, Goffredo D, Conti L, Di Febo F, Biella G, Toselli M et al. An optimized experimental strategy for efficient conversion of embryonic stem (ES)-derived mouse neural stem (NS) cells into a nearly homogeneous mature neuronal population. Neurobiol Dis 2009; 34: $320-331$.

20. Pool M, Thiemann J, Bar-Or A, Fournier AE. NeuriteTracer: a novel ImageJ plugin for automated quantification of neurite outgrowth. J Neurosci Methods 2008; 168: 134-139.

21. Jansen PJ, Lutjohann D, Thelen KM, von Bergmann K, van Leuven F, Ramaekers FC et al. Absence of $A p o E$ upregulates murine brain $A p o D$ and $A B C A 1$ levels, but does not affect brain sterol levels, while human ApoE3 and human ApoE4 upregulate brain cholesterol precursor levels. J Alzheimers Dis 2009; 18: 319-329.

22. Wahrle SE, Jiang H, Parsadanian M, Kim J, Li A, Knoten A et al. Overexpression of ABCA1 reduces amyloid deposition in the PDAPP mouse model of Alzheimer disease. J Clin Invest 2008; 118: 671-682.

23. Herz J, Bock HH. Lipoprotein receptors in the nervous system. Annu Rev Biochem 2002; 71 : 405-434.

24. Miserez AR, Cao G, Probst LC, Hobbs HH. Structure of the human gene encoding sterol regulatory element binding protein 2 (SREBF2). Genomics 1997; 40: 31-40.

25. Pfrieger FW. Role of glial cells in the formation and maintenance of synapses. Brain Res Rev 2010; 63: 39-46

26. Eroglu C, Barres BA. Regulation of synaptic connectivity by glia. Nature 2010; 468: 223-231.

27. Hayashi H, Campenot RB, Vance DE, Vance JE. Glial lipoproteins stimulate axon growth of central nervous system neurons in compartmented cultures. J Biol Chem 2004; 279 : 14009-14015. 
28. Goritz C, Thiebaut R, Tessier LH, Nieweg K, Moehle C, Buard I et al. Glia-induced neuronal differentiation by transcriptional regulation. Glia 2007; 55: 1108-1122.

29. Buard I, Steinmetz CC, Claudepierre T, Pfrieger FW. Glial cells promote dendrite formation and the reception of synaptic input in Purkinje cells from postnatal mice. Glia 2010; 58: 538-545

30. Fan QW, Yu W, Gong JS, Zou K, Sawamura N, Senda T et al. Cholesterol-dependent modulation of dendrite outgrowth and microtubule stability in cultured neurons. J Neurochem 2002; 80: 178-190.

31. Ilieva $\mathrm{H}$, Polymenidou M, Cleveland DW. Non-cell autonomous toxicity in neurodegenerative disorders: ALS and beyond. J Cell Biol 2009; 187: 761-772.

32. Tong J, Huang C, Bi F, Wu Q, Huang B, Liu X et al. Expression of ALS-linked TDP-43 mutant in astrocytes causes non-cell-autonomous motor neuron death in rats. EMBO J 2013; 32: 1917-1926.

33. Ballas N, Lioy DT, Grunseich C, Mandel G. Non-cell autonomous influence of MeCP2-deficient glia on neuronal dendritic morphology. Nat Neurosci 2009; 12 : 311-317.

34. Lioy DT, Garg SK, Monaghan CE, Raber J, Foust KD, Kaspar BK et al. A role for glia in the progression of Rett's syndrome. Nature 2011; 475: 497-500.

35. Di Malta C, Fryer JD, Settembre C, Ballabio A. Astrocyte dysfunction triggers neurodegeneration in a lysosomal storage disorder. Proc Natl Acad Sci USA 2012; 109: E2334-E2342.

36. Furman JL, Sama DM, Gant JC, Beckett TL, Murphy MP, Bachstetter AD et al. Targeting astrocytes ameliorates neurologic changes in a mouse model of Alzheimer's disease. $J$ Neurosci 2012; 32: 16129-16140.

37. Tong X, Ao Y, Faas GC, Nwaobi SE, Xu J, Haustein MD et al. Astrocyte Kir4.1 ion channel deficits contribute to neuronal dysfunction in Huntington's disease model mice. Nat Neurosci 2014 Mar: 30

38. Hughes EG, Elmariah SB, Balice-Gordon RJ. Astrocyte secreted proteins selectively increase hippocampal GABAergic axon length, branching, and synaptogenesis. Mol Cell Neurosci 2010; 43: 136-145.

39. Karasinska JM, Rinninger F, Lutjohann D, Ruddle P, Franciosi S, Kruit JK et al. Specific loss of brain ABCA1 increases brain cholesterol uptake and influences neuronal structure and function. J Neurosci 2009; 29: 3579-3589.

40. Jiang Q, Lee CY, Mandrekar S, Wilkinson B, Cramer P, Zelcer N et al. ApoE promotes the proteolytic degradation of Abeta. Neuron 2008; 58: 681-693.

41. Cermenati G, Brioschi E, Abbiati F, Melcangi RC, Caruso D, Mitro N. Liver $X$ receptors, nervous system, and lipid metabolism. J Endocrinol Invest 2013; 36. 435-443.

42. Futter M, Diekmann H, Schoenmakers E, Sadiq O, Chatterjee K, Rubinsztein DC. Wild-type but not mutant huntingtin modulates the transcriptional activity of liver $\mathrm{X}$ receptors. J Med Genet 2009; 46: 438-446.

43. Camargo N, Smit AB, Verheijen MH. SREBPs: SREBP function in glia-neuron interactions. FEBS J 2009; 276: 628-636.

44. Verheijen MH, Camargo N, Verdier V, Nadra K, de Preux Charles AS, Medard JJ et al. SCAP is required for timely and proper myelin membrane synthesis. Proc Natl Acad Sci USA 2009; 106: 21383-21388.
45. Camargo N, Brouwers JF, Loos M, Gutmann DH, Smit AB, Verheijen MH. High-fat diet ameliorates neurological deficits caused by defective astrocyte lipid metabolism. FASEB $J$ 2012; 26: 4302-4315.

46. Bjorkhem I. Do oxysterols control cholesterol homeostasis? J Clin Invest 2002; 110 725-730.

47. Cummings DM, Cepeda C, Levine MS. Alterations in striatal synaptic transmission are consistent across genetic mouse models of Huntington's disease. ASN neuro 2010; 2: $\mathrm{e} 00036$.

48. Milnerwood AJ, Raymond LA. Early synaptic pathophysiology in neurodegeneration: insights from Huntington's disease. Trends Neurosci 2010; 33: 513-523.

49. Orth M, Schippling S, Schneider SA, Bhatia KP, Talelli P, Tabrizi SJ et al. Abnormal motor cortex plasticity in premanifest and very early manifest Huntington disease. J Neurol Neurosurg Psychiatry 2010; 81: 267-270.

50. Valenza M, Leoni V, Tarditi A, Mariotti C, Bjorkhem I, Di Donato S et al. Progressive dysfunction of the cholesterol biosynthesis pathway in the R6/2 mouse model of Huntington's disease. Neurobiol Dis 2007; 28: 133-142.

51. Pfrieger FW. Role of cholesterol in synapse formation and function. Biochim Biophys Acta 2003; 1610: 271-280.

52. Christopherson KS, Ullian EM, Stokes CC, Mullowney CE, Hell JW, Agah A et al. Thrombospondins are astrocyte-secreted proteins that promote CNS synaptogenesis. Cell 2005; 120: 421-433.

53. Funfschilling U, Jockusch WJ, Sivakumar N, Mobius W, Corthals K, Li S et al. Critical time window of neuronal cholesterol synthesis during neurite outgrowth. J Neurosci 2012; 32 $7632-7645$.

54. Hulce JJ, Cognetta AB, Niphakis MJ, Tully SE, Cravatt BF. Proteome-wide mapping of cholesterol-interacting proteins in mammalian cells. Nature methods 2013; 10: 259-264.

55. Nieweg K, Schaller H, Pfrieger FW. Marked differences in cholesterol synthesis between neurons and glial cells from postnatal rats. J Neurochem 2009; 109: 125-134.

56. Ullian EM, Sapperstein SK, Christopherson KS, Barres BA. Control of synapse number by glia. Science 2001; 291: 657-661.

57. Sodero AO, Vriens J, Ghosh D, Stegner D, Brachet A, Pallotto M et al. Cholesterol loss during glutamate-mediated excitotoxicity. EMBO J 2012; 31: 1764-1773.

58. Taghibiglou C, Martin HG, Lai TW, Cho T, Prasad S, Kojic L et al. Role of NMDA receptordependent activation of SREBP1 in excitotoxic and ischemic neuronal injuries. Nat Med 2009; 15: 1399-1406.

59. Aono M, Lee Y, Grant ER, Zivin RA, Pearlstein RD, Warner DS et al. Apolipoprotein E protects against NMDA excitotoxicity. Neurobiol Dis 2002; 11: 214-220.

60. Paul SM, Doherty JJ, Robichaud AJ, Belfort GM, Chow BY, Hammond RS et al. The major brain cholesterol metabolite 24(S)-hydroxycholesterol is a potent allosteric modulator of N-methyl-D-aspartate receptors. J Neurosci 2013; 33: 17290-17300.

61. del Toro D, Xifro X, Pol A, Humbert S, Saudou F, Canals JM et al. Altered cholestero homeostasis contributes to enhanced excitotoxicity in Huntington's disease. J Neurochem 2010; 115: 153-167.

62. Colella AD, Chegenii N, Tea MN, Gibbins IL, Williams KA, Chataway TK. Comparison of stain-free gels with traditional immunoblot loading control methodology. Anal Biochem 2012; 430: 108-110. 